\title{
Continuous selections of multivalued mappings
}

Dušan Repovš and Pavel V. Semenov

\begin{abstract}
This survey covers in our opinion the most important results in the theory of continuous selections of multivalued mappings (approximately) from 2002 through 2012. It extends and continues our previous such survey which appeared in Recent Progress in General Topology, II which was published in 2002. In comparison, our present survey considers more restricted and specific areas of mathematics. We remark that we do not consider the theory of selectors (i.e. continuous choices of elements from subsets of topological spaces) since this topics is covered by another survey in this volume.
\end{abstract}

\section{Preliminaries}

A selection of a given multivalued mapping $F: X \rightarrow Y$ with nonempty values $F(x) \neq \emptyset$, for every $x \in X$, is a mapping $\Phi: X \rightarrow Y$ (in general, also multivalued) which for every $x \in X$, selects a nonempty subset $\Phi(x) \subset F(x)$. When all $\Phi(x)$ are singletons, a selection is called singlevalued and is identified with the usual singlevalued mapping $f: X \rightarrow Y,\{f(x)\}=\Phi(x)$. As a rule, we shall use small letters $f, g, h, \phi, \psi, \ldots$ for singlevalued mappings and capital letters $F, G, H, \Phi, \Psi, \ldots$ for multivalued mappings.

There exist a great number of theorems on existence of selections in the category of topological spaces and their continuous (in various senses) map-

Dušan Repovš

Faculty of Education and Faculty of Mathematics and Physics, University of Ljubljana, P. O. Box 2964, Ljubljana, Slovenia 1001

e-mail: dusan.repovs@guest.arnes.si

Pavel V. Semenov

Department of Mathematics, Moscow City Pedagogical University, 2-nd Sel'skokhozyastvennyi pr. 4, Moscow, Russia 129226

e-mail: pavels@orc.ru 
pings. However, the citation index of one of them is by an order of magnitude higher than for any other one: this is the Michael selection theorem for convexvalued mappings [67, Theorem 3.2", (a) $\Rightarrow(\mathrm{b})]$ :

Theorem 1. A multivalued mapping $F: X \rightarrow B$ admits a continuous singlevalued selection, provided that the following conditions are satisfied:

(1) $X$ is a paracompact space;

(2) $B$ is a Banach space;

(3) $F$ is a lower semicontinuous (LSC) mapping;

(4) For every $x \in X, F(x)$ is a nonempty convex subset of $B$; and

(5) For every $x \in X, F(x)$ is a closed subset of $B$.

Moreover, the reverse implication $(b) \Rightarrow(a)$ in $[67$, Theorem 3.2"] states that a $T_{1}$-space $X$ is paracompact whenever each multivalued mapping $F$ : $X \rightarrow B$ with properties $(2)-(5)$ above, admits a continuous singlevalued selection.

If one identifies a multivalued mapping $F: X \rightarrow Y$ with its graph $\Gamma_{F} \subset$ $X \times Y$ then the lower semicontinuity (LSC) of $F$ means exactly the openess of the restriction $\left.\pi_{1}\right|_{\Gamma_{F}}: \Gamma_{F} \rightarrow X$ of the projection $\pi_{1}: X \times Y \rightarrow X$ onto the first factor. In more direct terms, lower semicontinuity of a multivalued mapping $F: X \rightarrow Y$ between topological spaces $X$ and $Y$ means that the (large) preimage $F^{-1}(U)=\{x \in X: F(x) \cap U \neq \emptyset\}$ of any open set $U \subset Y$ is an open subset of the domain $X$. Applying the Axiom of Choice, we obtain:

Lemma 1. The following statements are equivalent:

(1) $F: X \rightarrow Y$ is a lower semicontinuous mapping;

(2) For each $(x ; y) \in \Gamma_{F}$ and each open neighborhood $U(y)$ there exists a local singlevalued (not necessarily continuous) selection of $F$, say $s: x^{\prime} \mapsto$ $s\left(x^{\prime}\right) \in F\left(x^{\prime}\right) \cap U(y)$, defined on some open neighborhood $V(x)$.

Therefore, the notion of lower semicontinuity is by definition related to the notion of selection. Symmetrically, if the (large) preimage $F^{-1}(A)=\{x \in$ $X: F(x) \cap A \neq \emptyset\}$ of any closed set $A \subset Y$ is a closed subset of the domain $X$, then the mapping $F: X \rightarrow Y$ is said to be upper semicontinuous (USC). Note that a more useful definition of upper semicontinuity of $F$ is that the (small) preimage $F_{-1}(U)=\{x \in X: F(x) \subset U\}$ of any open $U \subset Y$ is an open subset of the domain $X$.

Let us now reformulate the other three principal Michael's theorems on selections.

Theorem 2 ([68]). A multivalued mapping $F: X \rightarrow Y$ admits a continuous singlevalued selection, provided that the following conditions are satisfied:

(1) $X$ is a zero-dimensional (in dim-sense) paracompact space;

(2) $Y$ is a completely metrizable space;

(3) F is a LSC mapping; and 
(4) For every $x \in X, F(x)$ is a closed subset of $Y$.

Theorem 3 ([72]). A multivalued mapping $F: X \rightarrow Y$ admits a compactvalued USC selection $H: X \rightarrow Y$, which in turn, admits a compactvalued $L S C$ selection $G: X \rightarrow Y$ (i.e. $G(x) \subset H(x) \subset F(x), x \in X$ ), provided that the following conditions are satisfied:

(1) $X$ is a paracompact space;

(2) $Y$ is a completely metrizable space;

(3) $F$ is a LSC mapping; and

(4) For every $x \in X, F(x)$ is a closed subset of $Y$.

Theorem 4 ([68]). Let $n \in \mathbb{N}$. A multivalued mapping $F: X \rightarrow Y$ admits a continuous singlevalued selection provided that the following conditions are satisfied:

(1) $X$ is a paracompact space with $\operatorname{dim} X \leq n+1$;

(2) $Y$ is a completely metrizable space;

(3) F is a LSC mapping;

(4) For every $x \in X, F(x)$ is an n-connected subset of $Y$; and

(5) The family of values $\{F(x)\}_{x \in X}$ is equi-locally $n$-connected.

A resulting selection of a given multivalued mapping $F$ is practically always constructed as a uniform limit of some sequence of approximate selections. A typical difficult situation arises with the limit point (or the limit subset). Such a limit point (or a subset) can easily end up in the boundary of the set $F(x)$, rather than in the set $F(x)$, if one does not pay attention to a more careful construction of the uniform Cauchy sequence of approximate selections.

In general, for an arbitrary Banach space $B$, there exists a LSC mapping $F:[0 ; 1] \rightarrow B$ with convex (nonclosed) values and without any continuous singlevalued selections (cf. [67, Example 6.2] or [85, Theorem 6.1]). On the other hand, every convexvalued LSC mapping of a metrizable domain into a separable Banach space admits a singlevalued selection, provided that all values are finite-dimensional [67, Theorem 3.1"']. Another kind of omission of closedness was suggested in $[16,73]$. It turns out that such omission can be made over a $\sigma$-discrete subset of the domain.

An alternative to pointwise omission of closedness is to consider some uniform versions of such omission. Namely, one can consider closedness in a fixed subset $Y \subset B$ instead of closedness in the entire Banach space $B$. Due to a deep result of van Mill, Pelant and Pol [79], existence of selections under such assumption implies that $Y$ must be completely metrizable, or in other words, a $G_{\delta}$-subset of $B$.

Due to the Aleksandrov Theorem, each of Theorems 2-4 remains valid under a replacement of the entire completely metrizable range space by any of its $G_{\delta}$-subsets. However, what happens with Theorem 1 under such a substitution? What can one (informally) say concerning the links between 
the metric structure and the convex structure induced on a $G_{\delta}$-subset from the entire Banach space?

Thus, during the last two decades one of the most intriguing questions in the selection theory was the following problem:

Problem 1 ([74]). Let $Y$ be a convex $G_{\delta}$-subset of a Banach space $B$. Does then every LSC mapping $F: X \rightarrow Y$ of a paracompact space $X$ with nonempty convex closed values into $Y$ have a continuous singlevalued selection?

In the next section we shall present some (partial) affirmative answers, as well as the counterexample of Filippov [30, 31].

\section{Solution of the $G_{\delta}$-problem}

Summarizing the results below, the answer to the $G_{\delta}$-problem is affirmative for domains which are "almost" finite-dimensional, whereas the answer is negative for domains which are essentially infinite-dimensional, for example, for domains which contain a copy of the Hilbert cube.

For finite-dimensional domains $X$, the $G_{\delta}$-problem has an affirmative solution simply because the family of convex closed subsets of a Banach space is $E L C^{n}$ and every convex set is $C^{n}$ for every $n \in \mathbb{N}$. Hence Theorem 4 can be applied. For a finite-dimensional range $B$ and moreover, for all finitedimensional values closed in $Y \subset B$, the problem is also trivial, because one can use the compactvalued selection Theorem 3 and the fact that the closed convex hull of a finite-dimensional compact space coincides with its convex hull.

As for ways of uniform omission of closedness in the range space let us first consider the simplest case when $Y=G$ is a unique open subset of a Banach space $B$. Separately we extract the following well-known folklore result (it probably first appeared in an implicit form in Corson and Lindenstrauss $[20])$.

Lemma 2. (Localization Principle) Suppose that a convexvalued mapping $F: X \rightarrow Y$ of a paracompact domain $X$ into a topological vector space $Y$ admits a singlevalued continuous selection over each member of some open covering $\omega$ of the domain. Then $F$ admits a global singlevalued continuous selection.

Taking for any $x \in X$ and $y \in F(x) \subset G$, an arbitrary open ball $D$, centered at $y$, such that the closure $C \operatorname{los}(D)$ is a subset of $G$, and invoking the Localization Principle we obtain:

Lemma 3. Given any paracompact space $X$ and any open subset $G$ of a Banach space $B$, every LSC mapping $F: X \rightarrow G$ with nonempty convex 
values admits a singlevalued continuous selection, whenever all values $F(x)$ are closed in $G$.

Somewhat different approach can be obtained using the following:

Lemma 4. For any compact subset $K$ of a convex closed (in $G$ ) subset $C$ of an open subset $G$ of a Banach space $B$, the closed (in B) convex hull $C l o s(\operatorname{conv}(K))$ also lies in $C$.

Thus, as it was pointed out in $[74,88]$, one can affirmatively resolve the $G_{\delta^{-}}$ problem for an arbitrary intersection of countably many open convex subsets of $B$.

Lemma 5. Let $\left\{G_{n}\right\}, n \in \mathbb{N}$, be a sequence of open convex subsets of a $B a$ nach space and $F: X \rightarrow Y=\bigcap_{n} G_{n}$ a LSC mapping of a paracompact space $X$ with nonempty convex values. Then $F$ admits a singlevalued continuous selection, whenever all values $F(x)$ are closed in $Y$.

In fact, it suffices to pick a compactvalued LSC selection $H: X \rightarrow Y$ of the mapping $F$ (cf. Theorem 3). Then the multivalued mapping $C l o s(\operatorname{conv}(H))$ : $x \mapsto C \operatorname{los}(\operatorname{conv}(H(x)))$ is a selection of the given mapping $F$ and it remains to apply Theorem 1 to the LSC mapping $C l o s(\operatorname{conv}(H))$.

Michael and Namioka [77] characterized those convex $G_{\delta}$-subsets $Y \subset B$ which are stable with respect to taking closed convex hulls of compact subsets. Note that they essentially used the construction of Filippov's counterexample $[30,31]$.

Theorem 5 ([77]). Let $Y \subset B$ be a convex $G_{\delta}$-subset of a Banach space $B$. Then the following statements are equivalent:

(1) If $K \subset Y$ is compact then so is the closed (in $Y$ ) convex hull of $K$;

(2) For any paracompact space $X$, each LSC mapping $F: X \rightarrow Y$ with convex closed (in $Y$ ) values admits a continuous singlevalued selection;

(3) Same as (2) but with $X$ assumed to be compact and metrizable.

Moreover, they observed that Theorem 5 remains valid for nonconvex $Y$, provided that (1) is modified by also requiring that $K \subset C$ for some closed (in $Y$ ) convex subset $C \subset Y$. Hence, the equivalence $(2) \Leftrightarrow(3)$ of Theorem 5 holds for any $G_{\delta}$-subset $Y$ of a Banach space.

Returning to the restrictions for domains, recall that Gutev [35] affirmatively resolved the $G_{\delta}$-problem for domains $X$ which are either a countably dimensional metric space or a strongly countably dimensional paracompact space. In fact, he proved that in both cases under the hypotheses of the problem, the existence of a singlevalued continuous selection is equivalent to the existence of a compactvalued USC selection. The latter statement is true, because each domain of such type can be represented as the image of some zero-dimensional paracompact space under some closed surjection with all preimages of points being finite. 
In 2002, Gutev and Valov [43] obtained a positive answer for domains with the so-called $C$-property. They introduced a certain enlargement of the original mapping $F$. Roughly speaking, they defined $W_{n}(x)$ as the set of all $y \in Y=\bigcap_{n \in N} G_{n}$ which are closer to $F(x)$ than to $B \backslash G_{n}$. It turns out that each of the mappings $W_{n}$ has an open graph and all of its values are contractible.

Applying the selection theorem of Uspenskii [110] for $C$-domains, one can first find selections for each $W_{n}$ and then for the pointwise intersections $\bigcap W_{n}(x)$ (for details cf. [43]). Their technique properly works even for arbitrary (nonconvex) $G_{\delta}$-subsets $Y \subset B$. Unfortunately, such a method does not work outside the class of $C$-domains, because Uspenskii's theorem gives a characterization of the $C$-property.

This was the reason why we stated the following problem in our previous survey:

Problem 2 ([88]). Are the following statements equivalent:

(1) $X$ is a $C$-space.

(2) Each LSC mapping $F: X \rightarrow Y$ to a $G_{\delta}$-subset of $Y$ of a Banach space $B$ with convex closed (in $Y$ ) values admits a continuous singlevalued selection.

Karassev has resolved this problem for weakly infinite-dimensional compact domains.

Theorem 6 ([53]). Let $X$ be a compact Hausdorff space and suppose that property (2) above holds. Then $X$ is weakly infinite-dimensional.

Observe that the (non)coincidence of the classes of $C$-spaces and weakly infinite-dimensional spaces is one of the oldest and still unsolved problems in dimension theory. The advantage of compact spaces is that in this case there is a set of various criteria for weak infinite-dimensionality. In particular, Karassev [53] used the fact that a compact space $X$ is weakly infinite-dimensional if and only if for any mapping $f: X \rightarrow Q$ to the Hilbert cube there exists a mapping $g: X \rightarrow Q$ such that $f(x) \neq g(x)$, for all $x \in X$.

Ending with the affirmative answers, let us recall that in our previous survey we directly suggested (cf. p. 427 in [88]) the area for finding a counterexample. In fact, having Lemma 5 , one needs to find a convex $G_{\delta}$-subset $Y$ of a Banach space $B$, such that $Y$ is not an intersection of countably many open convex sets. Such a situation in fact, appeared in measure theory: for example in the compactum $P[0,1]$ of probability measures on the segment $[0,1]$ such is the convex complement of any absolutely continuous measure. To extract the main idea of Filippov's construction we introduce a temporary notion.

Definition 1. A convex compact subset $K$ of a Fréchet space $B$ has the Weizsäcker-Filippov property (WF-property) if there exists: 
(1) A proper convex $G_{\delta}$-subset $Y \subset K$ which contains the set $\operatorname{extr}(K)$ of all extreme points of $K$; and

(2) A LSC convexvalued mapping $R: K \rightarrow K$ such that $R(x) \cap \operatorname{extr}(K) \neq$ $\emptyset, x \in K$ and $R(\operatorname{conv} A)=\operatorname{conv} A$, for any finite subset $A \subset \operatorname{extr}(K)$.

Lemma 6. If $K \subset B$ has the WF-property then the mapping $F: K \rightarrow Y$ defined by $F(x)=C \operatorname{Cos}_{Y}(R(x) \cap Y), \quad x \in K$, is a counterexample to the $G_{\delta}$-problem.

Proof. All values $F(x)$ are nonempty because $R(x) \cap \operatorname{extr}(K) \neq \emptyset$ and $\operatorname{extr}(K) \subset Y$. Clearly, $F(x)$ are convex closed (in $Y$ ) sets. The mapping $x \mapsto R(x) \cap Y$ is LSC because $R$ is LSC and $Y$ is dense in $K$. Hence $F: K \rightarrow Y$ is LSC because pointwise-closure operator preserves lower semicontinuity.

Suppose to the contrary, that $f: K \rightarrow Y$ is a singlevalued continuous selection of $F$. Then $f(x)=x$, provided that $x$ is an extreme point. Moreover, if $x, y \in \operatorname{extr}(K)$ then $f([x, y]) \subset F([x ; y])=[x, y]$ and $f([x, y]) \supset[x, y]$, since $f(x)=x, f(y)=y$, and because of the continuity of $f$. Similarly, $f(\operatorname{conv}\{x, y, z\})=\operatorname{conv}\{x, y, z\}$ for each extreme points $x, y, z$, and so on. Hence, $f(\operatorname{conv}(\operatorname{extr}(K)))=\operatorname{conv}(\operatorname{extr}(K)) \subset K$ is a dense subset of $K$ and $f(K)$ is also dense in $K$. However, $f(K)$ is compact since it is the image of a compact set $K$ under the continuous mapping $f$. Therefore $f(K)=K$ which contradicts with $f(K) \subset Y$ and $Y \neq K$.

Theorem 7. (1) The space $P[0 ; 1]$ of all probability measures on $[0 ; 1]$ has the WF-property.

(2) In any Banach space there exists a convex compact subset $K$ with the WF-property.

Proof. By the Keller theorem, the convex compact space $P[0 ; 1]$ can be affinely embedded into the Hilbert space, hence into every Banach space with a Schauder basis (hence into every Banach space). Hence (1) implies (2).

In order to check (1), pick an arbitrary absolutely continuous measure $\mu \in K=P[0 ; 1]$, for example the Lebesgue measure. For every $m \in K \backslash\{\mu\}$, denote by $l_{m, \mu}$ the infinite ray from the point $m$ through the point $\mu$. Define the convex complement of $\mu$ by setting

$$
Y=\left\{m \in K \backslash\{\mu\}: \quad l_{m, \mu} \cap K=[m ; \mu]\right\} .
$$

Clearly $Y$ is convex. For every point $x \in[0 ; 1]$, the Dirac measure $\delta_{x}$ belongs to $Y$ because $(1-t) \delta_{x}+t \mu, t>1$ is not a probability measure. Hence $\operatorname{extr}(K) \subset$ $Y$. Next,

$$
Y=\bigcap_{n=1}^{\infty}\left\{m \in K:\left(1-n^{-1}\right) \cdot \delta_{x}+n^{-1} \cdot \mu \notin K\right\}
$$

and this is why $Y$ is a proper convex $G_{\delta}$-subset of $K$. 
Finally, define $R: K \rightarrow K$ by setting $R(m)=\left\{m^{\prime} \in K: \operatorname{supp}\left(m^{\prime}\right) \subset\right.$ $\operatorname{supp}(m)\}$, where supp denotes the support of the probability measure, i.e. the set of all points $x \in[0 ; 1]$ with the property that the value of the measure is positive over each neighborhood of the point. It is a straightforward verification that $R: K \rightarrow K$ is a LSC convexvalued mapping and that the equality

$$
R\left(\sum \lambda_{i} \cdot \delta_{x_{i}}\right)=\operatorname{conv}\left\{\delta_{x_{1}}, \ldots, \delta_{x_{n}}\right\}, \quad x_{i} \in[0 ; 1], \quad \lambda_{i} \geq 0, \sum \lambda_{i}=1,
$$

is evidently true.

A version of the construction was proposed in [91] which (formally) avoids any probability measures and works directly in the Hilbert cube $Q=[0 ; 1]^{\mathbb{N}}$. Here is a sketch:

- $X=\left\{x \in Q: x_{1}=1, x_{n}=x_{2 n}+x_{2 n+1}, n \in \mathbb{N}\right\}$;

- $Y=\left\{x \in X: \sup \left\{x_{n} z_{n}^{-1}: n \in \mathbb{N}\right\}=\infty\right\}$, where $z \in X$ is arbitrarily chosen so that $\lim _{n \rightarrow \infty} z(n)=0$ and $z(n)>0$ for all $n$; and

- $F(x)=\Phi(x) \cap Y$, where $\Phi: X \rightarrow X$ is defined by

$$
\Phi(x)=\left\{y \in X: y_{n}=0 \text { whenever } x_{n}=0\right\} .
$$

Let us temporarily say that natural numbers $2 n$ and $2 n+1$ are sons of the number $n$, which in turn, we shall call the father of such twins. Thus each natural number has exactly 2 sons, 4 grandsons, etc. and the natural partial order, say $\prec$, immediately arises on the set $\mathbb{N}$. With respect to $\prec$, the set $\mathbb{N}$ can be represented as a binary tree $T$ and every $x \in X$ is a mapping $x: T \rightarrow[0 ; 1]$ with $x_{1}=1, x_{n}=x_{2 n}+x_{2 n+1}, n \in \mathbb{N}$. In other words, each $x \in X$ defines some probability distribution on each $n$-th level of the binary tree $T$.

Hence even though all proofs in this construction can be performed directly in the Hilbert cube, the set $X$ is in fact, a "visualization" of the set of all probability measures of the Cantor set and details of the proof look similar to those above.

In conclusion, we mention the paper [32] which demonstrated the essentiality of the $G_{\delta}$-assumption for $Y$ in Theorem 5 of Michael and Namioka. Briefly, it was proved that for every countable $A \subset[0 ; 1]$, the set $Y=P_{A}=$ $\{\mu \in P[0 ; 1]: \operatorname{supp}(\mu) \subset A\}$ has the property (1) from Theorem 5 . Then by using sets of probability measures with various countable supports, the authors constructed a convex subset $Y \subset \mathbb{R}^{2} \times l_{2}$ with property (1) and without property (2) from Theorem 5 . Note that, as it was proved by V. Kadets, the property (1) from Theorem 5 is equivalent to the closedness of $Y \subset B$ being not only a convex set, but also a linear subspace (cf. [75, Proposition 5.1]). 


\section{Selections and extensions}

There are intimate relations between selections and extensions and typically they appear together: if $A \subset X$ and $f: A \rightarrow Y$ then $\widehat{f}: X \rightarrow Y$ is an extension of $f$ if and only if $\widehat{f}$ is a selection of multivalued mapping $F_{A}: X \rightarrow Y$ defined by setting $F_{A}(x)=\{f(x)\}, x \in A$, and $F_{A}(x)=Y$ otherwise. Thus as a rule, each fact concerning existence of singlevalued selections implies some result on extensions. In the other direction, many basic theorems (or some of their special cases) about extensions are special cases of some appropriate selection theorems.

However, extension theory is certainly not simply a subtheory of selection theory: specific questions and problems need specific ideas and methods. For example, selection Theorem 1 implies that every continuous map $f: A \rightarrow Y$ from a closed subset $A$ of a paracompact domain $X$ into a Banach space $B$ has a continuous extension $\widehat{f}: X \rightarrow B$ with $f(X) \subset C \operatorname{los}(\operatorname{conv}(f(A)))$. However, the Dugundji extension theorem states somewhat differently: every continuous map $f: A \rightarrow Y$ from a closed subset $A$ of a metric (or stratifiable) domain $X$ into a locally convex topological vector space $B$ has a continuous extension $\widehat{f}: X \rightarrow B$ with $f(X) \subset \operatorname{conv}(f(A))$. Besides the differences in assumptions and conclusions, these two "similar" theorems are proved by almost disjoint techniques: a sequential procedure of some approximations in the Michael selection theorem and a straightforward answer by a formula in Dugundji extension theorem. It seems that the only common point are continuous partitions of unity.

To emphasize the difference on a more nontrivial level, let us recall that for a wide class of nonlocally convex, completely metrizable, topological vector spaces it was proved in [26] that all such spaces are absolute retracts (with respect to all metrizable spaces), abbreviated as $A R^{\prime} s$. At the same time, at present there is no known example of a nonlocally convex, completely metrizable, topological vector space $E$ which can be successfully substituted instead of Banach (or Fréchet) spaces $B$ into the assumption of the Michael selection Theorem 1. In particular, Dobrowolski stated (private communication) the following:

Problem 3. Is the space $l_{p}, 0<p<1$, of all $p$-summable sequences of reals an absolute selector, i.e. is it true that for every paracompact space (metric space, compact space) and every LSC mapping $F: X \rightarrow l_{p}$ with convex closed values, there exists a continuous singlevalued selection of $F$ ?

During the last decade one of the most interesting facts concerning relations between selections and extensions was obtained by Dobrowolski and van Mill [27]. To explain their main results recall that $g: X \rightarrow Y$ is said to be an $\varepsilon$-selection of a multivalued mapping $F: X \rightarrow Y$ into a metric space $(Y ; d)$ if $\operatorname{dist}(g(x), F(x))<\varepsilon$. Dobrowolski and van Mill used the term $\epsilon$-near selection for the case when the strong inequality $\operatorname{dist}(g(x), F(x))<\varepsilon$ 
is replaced by $\operatorname{dist}(g(x), F(x)) \leq \varepsilon$. Clearly, for closedvalued mappings 0-near selections are exactly selections.

Definition 2. A convex subset $Y$ of a vector metric space $(E ; d)$ has the finite-dimensional selection property (resp. finite-dimensional near selection property) if for every metrizable domain $X$ and every LSC mapping $F: X \rightarrow$ $Y$ with all compact convex and finite-dimensional values $F(x) \subset Y, x \in X$, there exists a continuous singlevalued selection of $F$ (resp., for every $\varepsilon>0$ there exists a continuous singlevalued $\varepsilon$-near selection of $F$ ).

Combining 3.3, 4.1, 5.4 and 6.1 from [27] we formulate the following:

Theorem 8 ([27]). For any convex subset $Y$ of a vector metric (not necessarily, locally convex) space $(E ; d)$ the following statements are equivalent:

(1) $Y$ is an $A R$;

(2) $Y$ has the finite-dimensional near selection property.

As for a specific selection theorem we cite:

Theorem 9 ([27]). Let $Y$ be a convex subset of a vector metric (not necessarily locally convex) space $(E ; d)$. Then for every metrizable domain $X$ and every compactvalued and convexvalued LSC mapping $F: X \rightarrow Y$ with $\max \{\operatorname{dim} F(x): x \in X\}<\infty$, there exists a continuous singlevalued selection $f$ of $F$.

Continuous singlevalued selections $f$ of a given multivalued mapping $F$ are usually constructed as uniform limits of sequences of certain approximations $\left\{f_{\varepsilon}\right\}, \varepsilon \rightarrow 0$, of $F$. Practically all known selection results are obtained by using one of the following two approaches for a construction of $\left\{f_{n}=f_{\varepsilon_{n}}\right\}, \varepsilon_{n} \rightarrow 0$. In the first (and the most popular) one, the method of outside approximations, mappings $f_{n}$ are continuous $\varepsilon_{n}$-selections of $F$, i.e. $f_{n}(x)$ all lie near the set $F(x)$ and all mappings $f_{n}$ are continuous. In the second one, the method of inside approximations, $f_{n}$ are $\delta_{n}$-continuous selections of $F$, i.e. $f_{n}(x)$ all lie in the set $F(x)$, however $f_{n}$ are discontinuous.

We emphasize that Theorem 9 is proved by using the method of inside approximation. This is a rare situation: all previously known to us examples are $[10,70,78,85]$. However, for nonlocally convex range spaces it is an adequate approach since for such spaces $Y$ the intersections of convex subsets with balls are in general, nonconvex. Also, compactness is not preserved under the convex closed hull operation.

It is very natural to try to substitute $\operatorname{dim} F(x)<\infty, x \in X$, in Theorem 9 instead of $\max \{\operatorname{dim} F(x): x \in X\}<\infty$. It turns out that this is a futile attempt. Namely, 5.6 in [27] implies that Theorem 9 becomes false with such a change of the dimensional restriction.

Theorem 10 ([27]). There exist a linear metric vector space $E$ and a $L S C$ mapping $F: Q \rightarrow E$ from the Hilbert cube $Q$, such that $E$ contains the tower $\left\{E_{n}\right\}$ of closed subsets with the following properties: 
(1) $Q=\cup Q_{n}$, where $Q_{n}=F^{-1}\left(E_{n}\right)$;

(2) The restrictions $\left.F\right|_{Q_{n}}$ satisfy all assumptions of Theorem 9; and

(3) For arbitrary choices of continuous selections $f_{n}: Q_{n} \rightarrow E$ of $\left.F\right|_{Q_{n}}$ their pointwise limit $f=\lim _{n} f_{n}$ is not a continuous mapping, whenever such a pointwise limit exists.

Note that due to the Localization principle (Lemma 1) the assumption $\max \{\operatorname{dim} F(x): x \in X\}<\infty$ in Theorem 9 can be replaced by its local version $\max \{\operatorname{dim} F(x): x \in U(x)\}<\infty$ for some neighborhood $U(x)$ of $x$. Two slight generalizations of Theorem 9 were presented in [91]: in the first one $Y$ was replaced by a $G_{\delta}$-subset and in the second the closedness restriction for values $F(x)$ was omitted.

\section{Theorem 11 ([91]).}

(1) Let $F: X \rightarrow Y$ be a LSC convexvalued mapping of a paracompact domain $X$ into a $G_{\delta}$-subset $Y$ of a completely metrizable linear space $E$. Then $F$ admits a singlevalued continuous selection provided that the values $F(x)$ are closed in $Y$ and that for every $x \in X$ there exists a neighborhood $U(x)$ such that $\max \left\{\operatorname{dim} F\left(x^{\prime}\right): x^{\prime} \in U(x)\right\}<\infty$.

(2) Let $F: X \rightarrow E$ be a LSC convexvalued mapping of a metrizable domain $X$ into a completely metrizable linear space $E$. Then $F$ admits a singlevalued continuous selection provided that for every $x \in X$ there exists a neighborhood $U(x)$ such that $\max \left\{\operatorname{dim} F\left(x^{\prime}\right): x^{\prime} \in U(x)\right\}<\infty$.

It is interesting to note that the metrizability of the domain in Theorem $11(2)$ (in comparison with the paracompactness in (1)) is an essential restriction because the proof is based on the density selection theorem of Michael [69] which works exactly for metrizable spaces.

Under dimensional restrictions for the domain, not for the values of the mapping, van Mill [78, Cor.5.2] obtained the following:

Theorem 12 ([78]). Let $X$ be a locally finite-dimensional paracompact space and $Y$ a convex subset of a vector metric space. Then each LSC mapping $F: X \rightarrow Y$ with complete convex values admits a singlevalued continuous selection.

On the other hand, Example 5.3 [27] shows that in general, Theorem 12 does not hold for domains which are unions of countably many finitedimensional compacta. However, if in the assumptions of Theorem 12 one passes to $C$-domains (which look as approximately finite-dimensional spaces) then exact selections can be replaced by $\varepsilon$-selections [27, Theorem 6.3].

Theorem 13 ([27]). Let $X$ be a $C$-space and $Y$ a convex subset of a vector metric space. Then each LSC mapping $F: X \rightarrow Y$ with convex values admits a singlevalued continuous $\varepsilon$-selections for any $\varepsilon>0$. 
In a volumninous paper, Gutev, Ohta and Yamazaki [42] systematically used selections and extensions for obtaining the criteria for various kinds of displacement of a subset in the entire space. Recall that $A \subset X$ is $C$ embedded in $X$ (resp., $C^{*}$-embedded in $X$ ) if every continuous (resp., every bounded continuous) function $f: A \rightarrow \mathbb{R}$ has a continuous extension to entire $X$. Below are some of their typical results, Theorems 4.3, 4.6, and 6.1.

Theorem 14 ([42]). For a subset $A$ of $X$ the following statements are equivalent:

(1) $A$ is $C^{*}$-embedded in $X$;

(2) For every Banach space $B$, every continuous mapping $F: X \rightarrow B$ with compact convex values $F(x)$ and every continuous selection $g: A \rightarrow B$ of the restriction $\left.F\right|_{A}$, there is a continuous extension $f: X \rightarrow B$ of $g$ which is also a selection of $F$;

(3) The same as (2), but without convexity of $F(x)$ and without $f$ being a selection of $F$;

(4) For every cardinal $\lambda$, every continuous maps $g, h: X \rightarrow c_{0}(\lambda)$ with $g(a) \leq h(a), a \in A$, and every $f: A \rightarrow c_{0}(\lambda)$ which separates $\left.g\right|_{A}$ and $\left.h\right|_{A}$, there exists a continuous extension $\widehat{f}: X \rightarrow c_{0}(\lambda)$ of $f$.

Here $c_{0}(\lambda)$ denotes the Banach space of all mappings $x$ from $\lambda$ to the reals such that the sets $\{\tau<\lambda:|x(\tau)| \geq \varepsilon\}$ are finite for all $\varepsilon>0$. Note that $c_{0}(1)=\mathbb{R}$ and $c_{0}\left(\aleph_{0}\right)=c_{0}$.

Theorem 15 ([42]). For a subset $A$ of $X$ the following statements are equivalent:

(1) $A$ is $C$-embedded in $X$;

(2) For every Banach space $B$, every lower $\sigma$-continuous mapping $F: X \rightarrow$ $B$ with closed convex values $F(x)$ and every continuous selection $g: A \rightarrow B$ of the restriction $\left.F\right|_{A}$, there is a continuous extension $f: X \rightarrow B$ of $g$ which is also the selection of $F$;

(3) The same as (2), but without convexity of $F(x)$ and without $f$ being a selection of $F$;

(4) For every cardinal $\lambda$, every continuous $g_{n}, h_{n}: X \rightarrow c_{0}(\lambda), n \in \mathbb{N}$, with $\liminf g_{n}(a) \leq \limsup h_{n}(a), a \in A$, and every $f: A \rightarrow c_{0}(\lambda)$ which separates $\liminf g_{n}(a)$ and $\lim \sup h_{n}(a)$, there exists a continuous extension $\widehat{f}: X \rightarrow c_{0}(\lambda)$ of $f$.

Here lower $\sigma$-continuity of a multivalued map means that it is the pointwise closure of a union of countably many continuous compactvalued mappings. Yet another characterization of $C$-embeddability can be formulated via mappings into open convex subsets of a Banach spaces.

Theorem $16([42])$. For a subset $A$ of $X$ the following statements are equivalent: 
(1) $A$ is $C$-embedded in $X$;

(2) For every Banach space B, every open convex $Y \subset B$, every lower $\sigma$ continuous mapping $F: X \rightarrow C \operatorname{los}(Y)$ with closed convex values $F(x)$ and every continuous selection $g: A \rightarrow B$ of $\left.F\right|_{A}$ with $g^{-1}(Y)=A \cap F^{-1}(Y)$, there is a continuous extension $f: X \rightarrow B$ of $g$ which is also the selection of $F$ and $f^{-1}(Y)=F^{-1}(Y)$;

(3) The same as (2), but with continuous compactvalued mapping $F$.

One more recent paper in which selections and extensions are simultaneously studied is due to Michael [76]. Below we unify 3.1 and 4.1 from [76].

Theorem 17 ([76]). For a metrizable space $Y$ the following statements are equivalent:

(1) $Y$ is completely metrizable;

(2) For every paracompact domain $X$ and every LSC mapping $F: X \rightarrow Y$ with closed values, there exists a LSC selection $G: X \rightarrow Y$ with compact values;

(3) For every closed subset $A$ of a paracompact domain $X$ and every continuous $g: A \rightarrow Y$, there exists a LSC mapping $G: X \rightarrow Y$ with compact values which extends $g$;

(4) Similar to (2) but for USC selection $H: X \rightarrow Y$ with compact values;

(5) Similar to (3) but for USC extension $H: X \rightarrow Y$ with compact values.

The implication $(5) \Rightarrow(1)$ is true in a more general case, namely when $Y$ is Czech-complete and $X$ is a paracompact $p$-space [81, 83].

To finish the section we return once again to comparison of the Dugundji extension theorem and the Michael selection theorem. Arvanitakis [2] proposed a uniform approach to proving both of these theorems. He worked with paracompact $k$-domains. Recall that a Hausdorff space $X$ is called a $k$-space if closedness of $A \subset X$ coincides with closedness of $A \cap K$ for all compact $K \subset X$. Below, completness of a locally convex vector space $E$ means that the closed convex hull operation preserves the compactness of subsets in $E$. Next, $C(T ; E)$ denotes the vector space of all continuous mappings from a topological space $T$ into $E$, endowed by the topology of uniform convergence on compact subsets.

Theorem $18([2])$. Let $X$ be a paracompact $k$-space, $Y$ a complete metric space, $E$ a locally convex complete vector space, and $F: X \rightarrow Y$ a LSC mapping. Then there exists a linear continuous operator $S: C(Y ; E) \rightarrow$ $C(X ; E)$ such that

$$
S(f)(x) \in C \operatorname{los}(\operatorname{conv}(f(F(x)))), \quad f \in C(Y ; E), \quad x \in X .
$$

The proof is based on study of regular exaves (extensions/averagings) operators but without any explicit use of probability measures and Milyutin mappings. Recently, Valov [111] suggested a generalization of this theorem to 
the case of an arbitrary paracompact domain. He extensively exploited in its full force the technique of functors $P_{\beta}$ and $\widehat{P}$, averaging operators, Milyutin mappings, and so on. He also used the universality of the zero-dimensional selection Theorem 2, i.e. the fact obtained in [94] that Theorem 2 implies both Theorems 1 and 3 .

Theorem 19 ([111]). Let $X$ be a paracompact space, $Y$ a complete metric space and $F: X \rightarrow Y$ a LSC mapping. Then:

(1) For every locally convex complete vector space $E$ there exists a linear operator $S_{b}: C_{b}(Y ; E) \rightarrow C_{b}(X ; E)$ such that

$$
S(f)(x) \in C \operatorname{los}(\operatorname{conv}(f(F(x)))), \quad f \in C(Y ; E), \quad x \in X,
$$

and such that $S_{b}$ is continuous with respect to the uniform topology and the topology of uniform convergence on compact subsets;

(2) If $X$ is a $k$-space and $E$ is a Banach space then $S_{b}$ can be continuously extended (with respect to both topologies) to a linear operator $S$ : $C(Y ; E) \rightarrow C(X ; E)$ with the property that $S(f)(x) \in C \operatorname{los}(\operatorname{conv}(f(F(x))))$.

Therefore by taking $Y=E=B$ to be a Banach space, $F$ a mapping with closed convex values and $f=\left.i d\right|_{Y}$, one can see that $S(f)$ is a selection of $F$ : $S(i d)(x) \in C \operatorname{los}(\operatorname{conv}(F(x))), x \in X$.

Next, if $A$ is a completely metrizable closed subspace of $X, E$ a locally convex complete vector space, and $F=F_{A}$ a mapping defined by $F(x)=\{x\}, x \in A$, and $F(x)=A, x \in X \backslash A$, then we see that $S_{b}(f)(x) \in$ $C l o s(\operatorname{conv}(f(F(x))))$, for any $f \in C_{b}(A ; E)$ and hence $S_{b}(f)(x)=f(x)$, whenever $x \in A$. Therefore $S_{b}(f)$ is an extension of $f$. Thus the result is on the one hand stronger than the Dugundji theorem because $X$ can be nonmetrizable, but on the other hand it is weaker because $A$ should be completely metrizable and the result relates to $C_{b}(A ; E)$, not to $C(A ; E)$.

As a corollary, the Banach-valued version of the celebrated Milyutin theorem can be obtained:

Theorem 20 ([111]). Let $X$ be an uncountable compact metric space, $K$ the Cantor set and $B$ a Banach space. Then $C(X ; B)$ is isomorphic to $C(K ; B)$.

\section{Selection characterizations of domains}

Theorem 1 states that assumptions (1) - (5) imply the existence of selections of a multivalued mapping $F$. Conversely, assumptions (2)-(5) together with existence of selections imply the condition (1) that a domain $X$ is a paracompact space. In other words, Theorem 1 gives a selection characterization of paracompactness. By varying the types of the range Banach spaces $B$, types of families of convex subsets of $B$, types of continuity of $F$, etc. one 
can try to find a selection characterization of some other topological types of domains. Originally, Michael [67] found such types of characterization for normality, collectionwise normality, normality and countable paracompactness, and perfect normality. Below we concentrate on recent results in this direction.

Gutev, Ohta and Yamazaki [41] obtained selection characterizations for three classes of domains inside the class of all $\lambda$-collectionwise normal spaces. Recall that this property means that for each discrete family $\left\{F_{\gamma}\right\}_{\gamma \in \Gamma}$ of closed subsets with $|\Gamma| \leq \lambda$ there is a discrete family $\left\{G_{\gamma}\right\}_{\gamma \in \Gamma}$ of open sets such that $F_{\gamma} \subset G_{\gamma}$. Note that the equivalence of (1) and (2) in Theorem 21 was proposed by Michael [67] (cf. the discussion concerning the proofs in Ch. II of [85]). We also observe that (4) in Theorems 21-13 resembles the classical Dowker separation theorem.

Theorem 21 ([41]). Let $\lambda$ be an infinite cardinal. Then for any $T_{1}$-space $X$ the following statements are equivalent:

(1) $X$ is $\lambda$-collectionwise normal;

(2) For every Banach space $B$ of the weight less than or equal to $\lambda$ and every LSC mapping $F: X \rightarrow B$ whose values $F(x)$ are convex compacta, or $F(x)=B$, there exists a continuous singlevalued selection of $F$;

(3) Same as (2) but for the Banach space $B=c_{0}(\lambda)$;

(4) For every closed $A \subset X$ and every singlevalued $g, h: A \rightarrow c_{0}(\lambda)$ such that $g \leq h, g$ is upper semicontinuous, and $h$ is lower semicontinuous, there exists a singlevalued continuous $f: X \rightarrow c_{0}(\lambda)$ such that $\left.f\right|_{A}$ separates $g$ and $h$, i.e. $g \leq\left. f\right|_{A} \leq h$.

Theorem $22([41])$. Let $\lambda$ be an infinite cardinal. Then for any $T_{1}$-space $X$ the following statements are equivalent:

(1) $X$ is countably paracompact and $\lambda$-collectionwise normal;

(2) For every generalized $c_{0}(\lambda)$-space $B$ and every LSC mapping $F$ : $X \rightarrow B$ with values $F(x)$ being convex compacta, or $F(x)=B$, and with $|F(x)|>1, x \in X$, there exists a continuous singlevalued selection $f$ of $F$ such that $f(x)$ is not an extreme point of $F(x), x \in X$;

(3) Same as (2) but for the Banach space $B=c_{0}(\lambda)$;

(4) Same as (4) in Theorem 21 but with strong inequalities $g<h$ and $g<\left.f\right|_{A}<h$.

Theorem 23 ([41]). Let $\lambda$ be an infinite cardinal. Then for any $T_{1}$-space $X$ the following statements are equivalent:

(1) $X$ is perfectly normal and $\lambda$-collectionwise normal;

(2) For every generalized $c_{0}(\lambda)$-space $B$ and every LSC mapping $F: X \rightarrow$ $B$ with values $F(x)$ being convex compacta, or $F(x)=B$, there exists a continuous singlevalued selection $f$ of $F$ such that $f(x)$ is not an extreme point of $F(x)$, whenever $|F(x)|>1$;

(3) Same as (2) but for the Banach space $B=c_{0}(\lambda)$; 
(4) Same as (4) in Theorem 21 but with strong inequalities $g(x)<f(x)<$ $h(x)$ for all $x \in A$ with $g(x)<h(x)$.

One of the key ingredients of the proofs is the fact that for a closedvalued and convexvalued mapping $F$, a selection avoiding all extreme points exists provided that $F$ admits two families of local disjoint selections. Certainly Theorems 21-23 constitute a base for Theorems 14-16 above.

In [41] authors stated the following question: Do Theorems 22 and 23 remain valid if in (2) one replaces $c_{0}(\lambda)$-space by an arbitrary Banach space $B$ of weight less than or equal to $\lambda$ ? Yamauchi answered this question in the affirmative.

Theorem 24 ([117]). Let $\lambda$ be an infinite cardinal. Then for any $T_{1}$-space $X$ the following statements are equivalent:

(1) $X$ is countably paracompact and $\lambda$-collectionwise normal;

(2) For every Banach space $B$ of weight less than or equal to $\lambda$, every $L S C$ mapping $F: X \rightarrow B$ with values $F(x)$ being convex compacta, or $F(x)=B$ and with $|F(x)|>1, x \in X$, there exists a continuous singlevalued selection $f$ of $F$ such that $f(x)$ is not an extreme point of $F(x), x \in X$.

Passing to $\lambda$-paracompactness, the following was proved in [117, Theorem 8]:

Theorem 25. Let $\lambda$ be an infinite cardinal. Then for any $T_{1}$-space $X$ the following statements are equivalent:

(1) $X$ is normal and $\lambda$-paracompact;

(2) The same as (2) in Theorem 24 but with closed values $F(x)$.

Considering $\lambda=\aleph_{0}$ one observes that in the Michael selection criteria for $X$ being normal and countably paracompact one can assume that a selection $f$ always avoids extreme points of values of multivalued mapping $F$ with $|F(x)|>1, x \in X$.

Analogously, a domain $X$ is perfectly normal and $\lambda$-paracompact if and only if for every Banach space $B$ with $w(B) \leq \lambda$ and every LSC mapping $F: X \rightarrow B$ with convex closed values $F(x)$ (not necessarily with $|F(x)|>$ $1, x \in X)$ there exists a continuous singlevalued selection $f$ of $F$ such that $f(x)$ is not an extreme point of $F(x)$, whenever $|F(x)|>1$ (cf. [117]).

Before stating one more result recall that normality of a covering $\omega$ means an existence of a sequence $\omega_{1}=\omega, \omega_{2}, \omega_{3}, \ldots$ of coverings such that each $\omega_{n+1}$ is a strong star refinement of $\omega_{n}$ and that a space is called a $\lambda-P F$ normal space if each its point-finite open coverings is normal. Yamauchi [115] characterized the class of $\lambda-P F$-normal spaces.

Theorem 26 ([115]). Let $\lambda$ be an infinite cardinal. Then for any $T_{1}$-space $X$ the following statements are equivalent:

(1) $X$ is $\lambda$-PF-normal; 
(2) For every simplicial complex $K$ with cardinality less than or equal to $\lambda$, every simplex-valued LSC mapping $F: X \rightarrow|K|$ has a continuous singlevalued selection.

An analogue of Theorem 26 for dimensional-like properties was also given in [115].

Here $|K|$ stands for a geometric realization of $K$, for example in the Banach space $l_{1}\left(\operatorname{Vert}_{K}\right)$, where $V e r t_{K}$ is the set of vertices of $K$, and $|K|$ is endowed with the metric topology, induced by this embedding. In fact, the initial result here was a theorem of Ivanšić and Rubin [51] that every simplexvalued mapping $F: X \rightarrow|K|_{w}$ admits a selection provided that $F: X \rightarrow$ $|K|_{w}$ is locally selectionable, where $|K|_{w}$ denotes $|K|$ endowed with the weak topology.

Yamauchi [114] proposed selection criteria for classes of realcompact spaces, Dieudonné complete spaces and Lindelöf spaces. The starting point was the result of Blum and Swaminatham [12] on selection characterization of realcompactness in terms of the so-called $\mathcal{S}$-fixed LSC mapping into a locally convex topological vector space. To avoid specific notations we collect here only the results for Lindelöf spaces.

Theorem 27 ([114]). For any regular space $X$ the following statements are equivalent:

(1) $X$ is Lindelöf;

(2) For every completely metrizable space $Y$ and every closedvalued LSC mapping $F: X \rightarrow Y$ there exist compactvalued USC mapping $H: X \rightarrow Y$ and compactvalued LSC mapping $G: X \rightarrow Y$ such that $G(x) \subset H(x) \subset$ $F(x), x \in X$ and $H(X)=\bigcup\{H(x): x \in X\}$ is separable;

(3) For every Banach space $B$ and every LSC mapping $F: X \rightarrow B$ with closed convex values there exists a continuous singlevalued selection $f$ of $F$ with separable image $f(X)$.

Next, we refer to our previous survey on selections to cite the ChobanGutev-Nedev conjecture.

Problem 4 ([88]). For every $T_{1}$ space $X$ the following statements are equivalent:

(1) $X$ is countably paracompact and collectionwise normal;

(2) For every Hilbert space $H$ and every LSC mapping $F: X \rightarrow H$ with closed convex values there exists a continuous singlevalued selection $f$ of $F$.

The implication $(2) \Rightarrow(1)$ is a standard exercise, while $(1) \Rightarrow(2)$ was a hard problem. During the last decade, in a series of papers, Shishkov successfully resolved the problem step by step. Here is a short list of his results. First, he reduced the situation to the case of bounded mappings $F: X \rightarrow Y$, i.e. mappings with the bounded in $Y$ image $F(X)=\bigcup\{F(x): x \in X\}$. 
Theorem 28 ([102]). For every countably paracompact space $X$ and every normed space $Y$ the following statements are equivalent:

(1) For every LSC mapping from $X$ to $Y$ with closed convex values there exists a continuous singlevalued selection;

(2) Same as (1) but for bounded LSC mappings.

Next, he solved the problem in the case of the domain $X$ a $\sigma$-product of metric spaces [103] and extended LSC mappings with normal and countably paracompact domains over the Dieudonné completions of the domains [104]. Then he proved the conjecture for domains which are hereditarily "nice" [105]:

Theorem 29 ([105]). Let $X$ be a countably paracompact and hereditarily collectionwise normal space, $B$ a reflexive Banach space and $F: X \rightarrow B$ a LSC mapping with convex closed values. Then there exists a continuous singlevalued selection $f$ of $F$.

In [106] Shishkov worked with a paracompactness-like restriction on domain.

Theorem 30 ([106]). Let $X$ be a c-paracompact and collectionwise normal space, $B$ a reflexive Banach space, and $F: X \rightarrow B$ a LSC mapping with convex closed values. Then there exists a continuous singlevalued selection $f$ of $F$.

Note that $X$ from the last theorem can be a nonparacompact space, and that collectionwise normality plus (c)-paracompactness of domain is not in general, a necessary restriction for existence od selections (cf. Nedev's theorem for mappings over $\left.\omega_{1}[80]\right)$.

Then Shishkov [107] proved the following fact which, together with Theorem 28, finally resolved Choban-Gutev-Nedev problem.

Theorem 31 ([107]). Let $X$ be a collectionwise normal space, $H$ a Hilbert space and $F: X \rightarrow H$ a LSC mapping with convex closed and bounded values. Then there exists a continuous singlevalued selection $f$ of $F$.

We note that the proof essentially uses the geometric and analytical structure of a Hilbert space. Thus for reflexive range spaces the problem is still open.

We end the section by recent results of Gutev and Makala [40] who have suggested a characterization for classes of domains by using a controlled local improvement of $\varepsilon$-selections up to a genuine selection, rather than using exact selections.

Theorem 32 ([40]). Let $\lambda$ be an infinite cardinal. For any $T_{1}$-space $X$ the following statements are equivalent:

(1) $X$ is countably paracompact and $\lambda$-collectionwise normal; 
(2) For every Banach space $B$ with $w(B) \leq \lambda$, every LSC mapping $F: X \rightarrow B$ with values $F(x)$ being convex compacta, or $F(x)=B$, every continuous $\varepsilon: X \rightarrow(0 ;+\infty)$ and every $\varepsilon$-selection $f_{\varepsilon}: X \rightarrow B$

of $F$, there exists a continuous singlevalued selection $f$ of $F$ such that $\operatorname{dist}\left(f(x), f_{\varepsilon}(x)<\varepsilon(x), x \in X\right.$.

Similarly we have for normality:

Theorem 33 ([40]). For any $T_{1}$-space $X$ the following statements are equivalent:

(1) $X$ is countably paracompact and normal;

(2) Same as (2) in Theorem 32 but for separable Banach spaces.

Note that the starting point of proofs in [40] was the following:

Theorem 34 ([40]). For any Banach space B the following statements are equivalent:

(1) For every collectionwise normal domain $X$ and every LSC $F: X \rightarrow$ $B$ with values $F(x)$ being convex compacta, or $F(x)=B$ there exists a continuous selection $f$ of $F$;

(2) Same as (1) but without possibility of $F(x)=B$.

\section{Generalized convexities}

5.1. Roughly speaking, there exists an entire mathematical "universe" devoted to various generalizations and versions of convexity. In our opinion, even if one simply lists the titles of "generalized convexities" one will find as a minimum, nearly 20 different notions.

As for the specific situation with continuous selections perhaps two principal approaches are really used here. With the inner point of view, one starts by introducing some type of "convex hull" operation and defines a convex set as a set which is preserved by such an operation. Typical examples are Menger's metric convexity [66], Michael's convex and geodesic structures [70], Mägerl's paved spaces [62], Bielawski's simplicial convexity [11], Horvath's structures [47], Saveliev's convexity [98], etc.

With respect to outer constructions, convex sets are introduced by some list of axioms and then the convex hull conv $A$ of a set $A$ is defined as the intersection $\cap\{C: A \subset C, C$ is convex $\}$. Among examples are: Levy's abstract convexity [58], Jamison's convexity [52], van de Vel's topological convexity [112], decomposable sets [33, 85], and many others. The following notion was introduced by van de Vel [112].

Definition 3. A family $\mathcal{C}$ of subsets of a set $Y$ is called a convexity on $Y$ if it contains $\emptyset$ and $Y$, is closed with respect to intersections of arbitrary 
subfamilies and is closed with respect to unions of an arbitrary updirected subfamilies.

Van de Vel [112] proved a Michael's selection type theorem for LSC mappings into completely metrizable spaces $Y$ endowed with a convexity $\mathcal{C}$ which satisfies a set of assumptions such as compatibility with metric uniformity, compactness and connectedness of polytopes (i.e. convex hulls of finite sets), etc. One of the crucial restriction was the so-called Kakutani $S_{4}$-property which means that every pair of disjoint convex sets admits extensions up to two complementary convex sets (i.e. half-spaces). In the special issue of "Topology and Applications" entirely dedicated to 50-th anniversary of selection theory and to the 80-th anniversary of Ernest Michael, Horvath [48] proposed an approach which gives a selection theorem for convexities with the relative $S_{4}$-property.

Theorem $35([48])$. Let $(Y ; \mathcal{C})$ be a completely metrizable space with convexity, let all polytopes be compact and connected, and let Kakutani $S_{4}$-property hold on polytopes with respect to the induced convexities. Then every LSC mapping $F: X \rightarrow Y$ from a paracompact domain and with closed convex values admits a continuous singlevalued selection

He also added facts on selections with results on extensions, approximations and fixed points. In fact, compactness and connectedness of polytopes together with the Kakutani $S_{4}$-property imply homotopical triviality of polytopes and moreover, of all completely metrizable convex sets. Therefore the following Horvath's theorem generalizes the previous one.

Theorem $36([48])$. Let $(Y ; \mathcal{C})$ be a completely metrizable space with convexity for which all polytopes are homotopically trivial. Then every LSC mapping $F: X \rightarrow Y$ with paracompact domain and with closed convex values admits a continuous singlevalued selection

The key technical ingredient proposed in [48] was the van de Vel property, which roughly speaking, fixes the existence of enough reflexive relations (entourages) $R \subset Y \times Y$ such that for every subset $Z \subset Y$ all simplicial complexes

$$
S_{R}(Z)=\left\{A \text { is a finite subset of } Z: Z \cap\left(\cap_{a \in A} R(a)\right) \neq \emptyset\right\}
$$

are homotopically trivial.

In the same issue of Topology and its Applications, Gutev [38] presented results on a somewhat similar matter. Briefly, he proposed another approach to proving the van de Vel selection theorem. He incorporated the proof into the technique of the so-called c-structures which was suggested around 1990 by Horvath [47]. A $c$-structure $\chi$ on a space $Y$ associates to every finite subset $A \subset Y$ some contractible subset $\chi(A) \subset Y$ such that $A \subset B$ implies $\chi(A) \subset \chi(B)$. In the case of the finite subsets $A$ of some prescribed $S \subset Y$ 
Gutev use the term c-system on $S$. Of course, as usual $\chi(\{y\})=\{y\})$. Perhaps the typical statements in [38] are, for example:

Theorem 37 ([38]). Let $X$ be a paracompact space, $Y$ a space, $\chi: F i n(S) \rightarrow$ $Y$ a c-system on $S \subset Y$, and $G: X \rightarrow S$ a Browder mapping (i.e. a multivalued mapping with all point-preimages open). Then $\operatorname{conv}_{\chi}(G)$ has a continuous singlevalued selection.

Theorem 38 ([38]). Let $X$ be a paracompact space, $(Y, \mu, \mathcal{C})$ a uniform space endowed with a $S_{4}$ convexity for which all polytopes are compact and all convex sets are connected. Let $\mathcal{V} \in \mu$ be an open convex cover of $X$ and $F: X \rightarrow Y$ a convexvalued LSC mapping. Then $F$ admits a LSC convexvalued selection $\Phi: X \rightarrow Y$ such that the family $\{\Phi(x)\}_{x \in X}$ of its values refines $\mathcal{V}$.

By using the latter fact, under the assumptions of the van de Vel theorem, one can construct a decreasing sequence $\Phi_{n}: X \rightarrow Y$ of convexvalued LSC mappings with $\sup _{x \in X} \operatorname{diam}\left(\Phi_{n}(x)\right) \rightarrow 0, n \rightarrow \infty$. Therefore the pointwise passing to $\cap_{n}\left\{\operatorname{Clos}\left(\Phi_{n}(x)\right)\right\}$ gives the desired selection of $F=\Phi_{0}$.

5.2. Some results on selections appeared for hyperconvex range spaces. Recall, that a metric space is hyperconvex if and only if it is injective with respect to extensions which preserves the modulus of continuity. In more direct terms, a metric space $(Y, d)$ is hyperconvex if and only if for every family $\left\{\left(y_{\alpha} ; r_{\alpha}\right) \in Y \times(0 ;+\infty)\right\}_{\alpha \in I}$ the inequalities $d\left(y_{\alpha}, y_{\beta}\right) \leq r_{\alpha}+r_{\beta}$ for all $\alpha, \beta \in I$ imply the nonemptiness of the intersection $\cap_{\alpha} D\left[y_{\alpha} ; r_{\alpha}\right]$ of closed balls $D\left[y_{\alpha} ; r_{\alpha}\right]=\operatorname{Clos}\left(D\left(y_{\alpha} ; r_{\alpha}\right)\right)$.

Each hyperconvex space admits the natural ball convexity in which polytopes are exactly the sets $\cap\{D[y, r]: A \subset D[y, r]\}$ with finite $A \subset Y$. Some authors $[64,121]$ also use the term sub-admissible for sets which are convex with respect to the ball convexity. For example, $\mathrm{Wu}[121$, Theorem 2.4] proved a selection theorem for the so-called locally uniform weak LSC mappings into hyperconvex spaces. Note that hyperconvex space equipped with the ball convexity is a uniform convex space with homotopically trivial polytopes, [48]. This is why a selection theorem for LSC mappings [121, Theorem 2.3] is a special case of selection theorems for generalized convexities. Markin [64] generalized Wu's result to a wider class of multivalued mappings which he named quasi $L S C$ although this is exactly the class of almost $L S C$ mappings introduced by Deutsch and Kenderov [24].

Another type of selection theorems for hyperconvex range spaces deals with various Lipschitz-type restrictions on a mappings and selections. A subset $Z \subset(Y ; d)$ is said to be externally hyperconvex if for any points $x_{\alpha} \in Z$ and any reals $r_{\alpha}$ with $d\left(x_{\alpha}, Z\right) \leq r_{\alpha}$ and $d\left(x \alpha, x_{\beta}\right) \leq r_{\alpha}+r_{\beta}$, the intersection $\cap_{\alpha} D\left[x_{\alpha}, r_{\alpha}\right] \cap Z$ is nonempty. Khamsi, Kirk and Yanez [54] proved the following:

Theorem 39 ([54]). Let $(Y ; d)$ be any hyperconvex space, $S$ any set, and $F: S \rightarrow Y$ any mapping with externally hyperconvex values. Then there exists a singlevalued selection $f$ of $F$ such that 


$$
d(f(x), f(y)) \leq \operatorname{Hausd}_{d}(F(x), F(y)) \quad x, y \in S .
$$

In particular, for a metric domain $S=(M ; \rho)$ and for a nonexpansive $F: S \rightarrow Y$, one can assume a selection $f$ of $F$ to be also nonexpansive, provided that all values $F(x)$ are bounded externally hyperconvex sets.

These results were applied by Askoy and Khamsi [3] for range spaces which are metric trees. Briefly, a metric tree is a metric space $(Y, d)$ such that for any $x, y \in Y$ there exists a unique arc joining $x$ and $y$ and such that the arc is isometric to a segment on the real line.

Theorem 40 ([3]). Let $(Y ; d)$ be a metric tree and $F: Y \rightarrow Y$ a mapping all of whose values all are bounded closed convex sets. Then there exists a singlevalued selection $f$ of $F$ such that

$$
d(f(x), f(y)) \leq \text { Hausd }_{d}(F(x), F(y)) x, y \in Y .
$$

A somewhat similar result was proved by Markin [65, Theorem 4.3]:

Theorem $41([65])$. Let $X$ be a paracompact space, $(Y ; d)$ a complete metric tree and $F: X \rightarrow Y$ an almost LSC mapping all of whose values are bounded closed convex sets. Then there exists a singlevalued continuous selection $f$ of F.

Selection theorems with respect to various types of convexities, $L$-structures, and $G$-structures, were obtained in $[25,59,118]$, etc. As a rule, all results here are special cases or versions of van de Vel's convexities, or Horvath's convexities.

5.3. As for some other "inner convexities", in a series of papers de Blasi and Pianigiani studied multivalued mappings into so-called $\alpha$-convex metric spaces $(Y ; d)$. This means the existence a continuous mapping $\alpha: Y \times Y \times$ $[0 ; 1] \rightarrow Y$ with natural restrictions

$$
\alpha(y, y, t)=y, \alpha(y, z, 0)=y, \alpha(y, z, 1)=z
$$

and with assumption that for some suitable $r=r(\alpha)>0$ and for every $\varepsilon<r$ there exists $0<\delta \leq \varepsilon$ such that the inequality

$$
\operatorname{Hausd}\left(\{\alpha(y, z, t): t \in[0 ; 1]\},\left\{\alpha\left(y^{\prime}, z^{\prime}, t\right): t \in[0 ; 1]\right\}\right)<\varepsilon
$$

for Hausdorff distance between curvilinear segments holds for each $\left(y, y^{\prime}\right) \in$ $Y^{2},\left(z, z^{\prime}\right) \in Y^{2}$ with $d\left(y, y^{\prime}\right)<\varepsilon$ and $d\left(z, z^{\prime}\right)<\delta$. Clearly, the last assumption reminds one of the estimate for $d\left(\alpha(y, z, t), \alpha\left(y^{\prime}, z^{\prime}, t\right)\right)$ from Michael's geodesic structure [70]. As usual, $C \subset Y$ is convex (with respect to $\alpha: Y \times Y \times[0 ; 1] \rightarrow Y)$ if $\{\alpha(y, z, t): t \in[0 ; 1]\} \subset C$ provided that $y \in C$ and $z \in C$.

Theorem 42 ([23]). Let $X$ be a paracompact space and $Y$ an $\alpha$-convex complete metric space. Then every LSC mapping $F: X \rightarrow Y$ with closed convex values admits a continuous singlevalued selection 
As it was shown earlier in [22] for the case of compact $X$ and $Y$, and $\operatorname{dim} X<\infty$, Theorem 43 is true for every $\alpha: Y \times Y \times[0 ; 1] \rightarrow Y$ with $\alpha(y, y, t)=y, \alpha(y, z, 0)=y, \alpha(y, z, 1)=z$. A set of applications in fixed point theory and in degree theory are also presented in [21, 23]. Kowalska [57] proved a theorem which unifies selection Theorem 42 with a graphapproximation theorems in the spirit of Ben-El-Mechaiekh and Kryszewski [9] who considered the case of classical convexity of a Banach space.

Among others results let us mention the paper of Kisielewicz [55] in which he used a convexity structures in functional Banach spaces $C\left(S, R^{n}\right)$ and $L^{\infty}\left(T, R^{n}\right)$ of all continuous mappings over a compact Hausdorff domain $S$ and all equivalence classes of almost everywhere bounded mappings over a measure space $(T, \mu)$. Both of these convexities remind of the notion of decomposable set of functions $[33,85]$.

Definition 4. (1) A subset $E \subset L^{\infty}\left(T, R^{n}\right)$ is said to be decomposable if $\chi_{A} f+\chi_{T \backslash} g$ belongs to $E$ provided that $f \in E, g \in E$ and $A$ is a measurable subset of $T$.

(2) A subset $E \subset L^{\infty}\left(T, R^{n}\right)$ is said to be $L$-convex if $p f+(1-p) g$ belongs to $E$ provided that $f \in E, g \in E$ and $p: T \rightarrow[0 ; 1]$ is a measurable function.

(3) A subset $E \subset C\left(S, R^{n}\right)$ is said to be $C$-convex if $h f+(1-h) g$ belongs to $E$ provided that $f \in E, g \in E$ and $h: T \rightarrow[0 ; 1]$ is a continuous function.

\section{Theorem 43 ([55]).}

(1) Let $X$ be a paracompact space and $F: X \rightarrow C\left(S, R^{n}\right)$ a LSC mapping with closed $C$-convex values. Then $F$ admits a continuous singlevalued selection if and only if its $n$-th derived mapping $F^{(n)}$ has nonempty values.

(2) Same as (1) but for $F: X \rightarrow L^{\infty}\left(T, R^{n}\right)$ with closed $L$-convex values.

Note that for closed subsets of $L^{\infty}\left(T, R^{n}\right)$ their $L$-convexity coincides with decomposability plus usual convexity [55, Proposition 4]. Recall that the derived mapping $F^{(1)}(x)$ of a multivalued mapping $F: X \rightarrow Y$ is defined by setting

$$
F^{(1)}(x)=\left\{y \in F(x):\left(x^{\prime} \rightarrow x\right) \Rightarrow \operatorname{dist}\left(y, F\left(x^{\prime}\right)\right) \rightarrow 0\right\} \subset F(x), x \in X
$$

and $F^{(k+1)}(x)=\left(F^{(k)}\right)^{(1)}(x)$. Also, a well-known result of Brown [14] states that for a convexvalued map $F: X \rightarrow R^{n}$, the nonemptiness of all $F^{(n)}(x), x \in X$, implies that $F^{(n)}: X \rightarrow R^{n}$ is a LSC selection of $F$. Hence the standard selection techniques can be applied to $F^{(n)}$. Rather simple examples show the essentiality of the finite-dimensionality of the range space.

5.4. Based on the ingenious idea of Michael who proposed in [71] the notion of a paraconvex set, the authors in $[84,86,87,89,99,100]$ systematically studied another approach to weakening (or, controlled omission) of convexity. Roughly speaking, to each closed subset $P \subset B$ of a Banach space one associates a numerical function, say $\alpha_{P}:(0,+1) \rightarrow[0,2)$, the so-called function 
of nonconvexity of $P$. The identity $\alpha_{P} \equiv 0$ is equivalent to the convexity of $P$ and the more $\alpha_{P}$ differs from zero the "less convex" is the set $P$.

Definition 5. The function of nonconvexity $\alpha_{P}(\cdot)$ of the set $P$ associates to each number $r>0$ the supremum of the set

$$
\left\{\sup \left\{\operatorname{dist}(q, P) / r: q \in \operatorname{conv}\left(P \cap D_{r}\right)\right\}\right\}
$$

over all open balls $D_{r}$ of the radius $r$.

For a function $\alpha:(0 ;+\infty) \rightarrow(0 ;+\infty)$ a nonempty closed subset $P$ of a Banach space is said to be $\alpha$-paraconvex provided that function $\alpha(\cdot)$ pointwisely majorates the function of nonconvexity $\alpha_{P}(\cdot)$. Then $P$ is said to be paraconvex provided that $\sup \alpha_{P}(\cdot)<1$.

In [89] we proved a paraconvex version of the Ky Fan-Sion minimax theorem.

Theorem 44 ([89]). Let $\alpha:(0, \infty) \rightarrow(0,1)$ be a function with the right upper limits less than 1 over the closed ray $[0, \infty)$. Let $f: X \times Y \rightarrow \mathbb{R}$ be a real valued function on Cartesian product of two AR-subcompacta $X$ and $Y$ of a Banach spaces and suppose that:

(1) For each $c \in \mathbb{R}$ and each $x_{0} \in X$ the set $\left\{y \in Y: f\left(x_{0}, y\right) \leq c\right\}$ is $\alpha$-paraconvex compact; and

(2) For each $d \in R$ and each $y_{0} \in Y$ the set $\left\{x \in X: f\left(x, y_{0}\right) \geq d\right\}$ is $\alpha$-paraconvex compact for a fixed $\alpha:(0, \infty) \rightarrow[0,1)$. Then

$$
\max _{X}\left(\min _{Y} f(x, y)\right)=\min _{Y}\left(\max _{X} f(x, y)\right) .
$$

It is interesting to note that our minimax theorem includes cases when finite intersections

$$
\bigcap_{i=1}^{n}\left\{\left\{x \in X: f\left(x, y_{i}\right) \geq c\right\}: y_{i} \in Y\right\}, \quad \bigcap_{j=1}^{k}\left\{\left\{y \in Y: f\left(x_{j}, y\right) \leq d\right\}: x_{j} \in X\right\}
$$

of sublevel and uplevel sets are possibly nonconnected: intersection of two paraconvex sets can be nonconnected.

Usually a proof of a minimax theorem for generalized convexities exploits the intersection property of convex sets and reduces minimax theorem to some kind of KKM-principle. In our case we used the fact that the closure of unions of directly ordered family of arbitrary paraconvex sets are also paraconvex. Therefore as a base for obtaining minimax theorem we have used the selection theory of multivalued mappings instead of versions of the KKM-principle.

In [93] we examined the following natural question: Does paraconvexity of a set with respect to the classical convexity structure coincide with convexity under some generalized convexity structure? In other words, is paraconvexity 
a real nonconvexity, or is it maybe a kind of some generalized convexity? It turns out that sometimes the answer is affirmative.

Theorem 45 ([93]). Let $0 \leq \alpha<0,5$ and $F: X \rightarrow B$ be a continuous multivalued mapping of a paracompact space $X$ into a Banach space $B$ such that all values $F(x)$ are bounded $\alpha$-paraconvex sets. Then there exists a continuous singlevalued mapping $\mathfrak{F}: X \rightarrow C_{b}(B, B)$ such that for every $x \in X$ the mapping $\mathfrak{F}_{x}: B \rightarrow B$ is a continuous retraction of $B$ onto the value $F(x)$ of $F$.

Here $C_{b}(B, B)$ denotes the Banach space of all continuous bounded mappings of a Banach space $B$ into itself. The key point of the proof is that the set $U \operatorname{Retr}_{P} \subset C_{b}(B, B)$ of all uniform retractions onto an $\alpha$-paraconvex set $P \subset B$ is a $\frac{\alpha}{1-\alpha}$-paraconvex subset of $C_{b}(B, B)$.

As a corollary, by continuously choosing a retraction onto a paraconvex sets, we showed that if in addition all values $F(x), x \in X$, are pairwise disjoint then the metric subspace $Y=\bigcup_{x \in X} F(x) \subset B$ admits a convex metric structure $\sigma$ such that each value $F(x)$ is convex with respect to $\sigma$.

Finally, let us mentioned the result of Makala [63, Theorem 3.1] who obtained the selection theorem for LSC mappings $F: X \rightarrow Y$ from a collectionwise normal domains $X$ such that each value $F(x)$ equals to $Y$ or, is a compact paraconvex subsets of $Y$. The main difficulty here was that the class of such values in general, is not closed with respect to intersections with balls.

5.5. Yet another type of a controlled "nonconvexity" which is in some sense intermediate between paraconvexity and Menger's metric convexity goes back to Vial [113] and during the last decade was intensively studied in [5, 49, 50].

For every two points $x$ and $y$ of a normed space $(Y ;\|\cdot\|)$ and for every $R \geq 0,5\|x-y\|$ denote by $D_{R}[x ; y]$ the intersection of all closed $R$-balls containing $x$ and $y$. Clearly, when $R \rightarrow+\infty$, such set $D_{R}[x ; y]$ tends (with respect to the Hausdorff distance) to the usual segment $[x ; y]$.

Definition $6([\mathbf{1 1 3}])$. A subset $A$ of a normed space $(Y ;\|\cdot\|)$ is said to be weakly convex w.r.t $R>0$ if for every $x, y \in A$, with $0<\|x-y\|<2 R$, there exists a point $z \in A \cap D_{R}[x ; y]$ that differs from $x$ and $y$.

In a Hilbert space $H$ the metric projection $P_{A}$ of an $R$-neighborhood of a weakly convex w.r.t. $R$ set $A$ is singlevalued. The set $\left\{(x ; y) \in \mathbb{R}^{2}: x \geq\right.$ 0 or $y \geq 0\}$ is $(\sqrt{2} / 2-)$ paraconvex but is not weakly convex with respect to arbitrary $R>0$. The set $\left\{x \in \mathbb{R}^{n}:\|x\| \geq R\right\}$ is not paraconvex and is weakly convex w.r.t $R$. However, sometimes weak convexity implies paraconvexity. For example, in a Hilbert space $H$, if $z \in H$ and $0<r<R$, then every weakly convex (w.r.t. $R$ ) subset $A \subset D(z ; r)$ is $(r / R)$-paraconvex [49].

Theorem 46 ([50]). Let $X$ be a paracompact space and $0<\varepsilon<R$. Then for every continuous singlevalued $\varepsilon$-selection $f_{\varepsilon}: X \rightarrow H$ of a LSC mapping $F: X \rightarrow H$ with closed and weakly convex (w.r.t. $R$ ) values there exists a continuous singlevalued selection. 
Theorem 47 ([50]). Let $X$ be a paracompact subset of a topological vector space $Z$ and a uniformly functional contractible subset of $Z$. Then for every $R>0$, each Hausdorff uniformly continuous mapping $F: X \rightarrow H$ with closed and weakly convex (w.r.t. $R$ ) values admits a continuous singlevalued selection.

Both contractibility and uniform continuity restrictions are essential as Examples 1-3 from [50] show.

5.6. To complete this section we mention one more nontrivial convexity structure. Namely, the so called tropical (or, max-plus) geometry in $(\mathbb{R} \cup$ $\{-\infty\}^{n}$. It is very intensively studied area with many various applications in abstract convex analysis, algebraic geometry, combinatorics, phylogenetic analysis, etc. For a survey and details cf. [120].

Definition 7. For an ordered $N$-tuple $t=\left(t_{j}\right)$ of "numbers" $t_{j} \in[-\infty ; 0]$ with $\max \left\{t_{j}\right\}=0$ and for a points $A_{1}\left(x_{1}^{1}, x_{2}^{1}, \ldots, x_{n}^{1}\right), \ldots, A_{N}\left(x_{1}^{N}, x_{2}^{N}, \ldots ., x_{n}^{N}\right)$ from $(\mathbb{R} \cup\{-\infty\})^{n}$ their max-plus $t$-combination is defined as the point $\left(\max _{j}\left\{x_{1}^{j}+t_{j}\right\}, \max _{j}\left\{x_{2}^{j}+t_{j}\right\} \ldots, \max _{j}\left\{x_{n}^{j}+t_{j}\right\}\right)$. A subset $C \subset(\mathbb{R} \cup\{-\infty\})^{n}$ is said to be max-plus convex if it contains all max-plus $t$-combinations of all of its points.

Zarichnyi proved a selection theorem for max-plus convexvalued mappings.

Theorem 48 ([122]). Let $X$ and $Y$ be compact metrizable spaces and $Y \subset$ $\mathbb{R}^{n}$. Then every LSC mapping $F: X \rightarrow Y$ with max-plus convex values admits a continuous singlevalued selection.

It is interesting to observe that the proof never uses any sequential procedure of approximation. Instead, Zarichnyi constructs a version of Milyutin surjection $M: Z \rightarrow X$ of a zero-dimensional compact space $Z$ onto $X$ and associating map $m: X \rightarrow I(Z)$ with values in idempotent probability measures. Next, exactly as in [94], the desired selection $f$ of $F$ is defined as the idempotent barycenter $f(x)=\int_{M^{-1}(x)} s(t) d m(x)$ for a suitable selection $s$ of the composition $F \circ M$ (such a selection exists due to Theorem 2).

\section{Multivalued selections}

The foundation for results of this section is the compactvalued selection Theorem 3. Historically there were various ways to prove this result or its variants: the original Michael's approach [72] via pointwise closures of limit point sets of certain "tree" of $2^{-n}$-singlevalued selections, Choban's method of coverings [16] which axiomatized and transformed Michael's construction into a maximally possible general form, approach based on the notion of (complete) sieves [18], and a proof via the 0-dimensional selection theorem [85]. 
In a series of papers Gutev recently proposed a more advanced point of view for sieves on a set $X$. Recall that a tree is a partially ordered set $(T ; \preceq)$ with all well-ordered sublevel sets $\{a: a \preceq b, a \neq b\}_{b \in T}$. Roughly speaking, in [37] a sieve on $X$ is defined as some kind of multivalued mapping $(T ; \preceq) \rightarrow X$ which is order-preserving with respect to inverse inclusion. In particular, as a corollary of his techniques, Gutev [37, Corollary 7.3] obtained the following generalization of compactvalued selection Theorem 3 , which was proposed earlier in [1].

Theorem 49 ([37]). A multivalued mapping $F: X \rightarrow Y$ admits a compactvalued USC selection $H: X \rightarrow Y$, which, in turn, admits a compactvalued LSC selection $G: X \rightarrow Y$, provided that the following conditions are satisfied:

(1) $X$ is a paracompact space;

(2) $Y$ is a monotonically developable and sieve-complete space;

(3) $F$ is a LSC mapping; and

(4) For every $x \in X, F(x)$ is a closed subset of $Y$.

Here, in comparison with compactvalued selection Theorem 3, only the restriction (2) is changed. Monotonically developable spaces are a natural generalization of Moore spaces. Note that $Y$ is monotonically developable and sieve-complete space if and only if $Y$ is the image of a completely metrizable non-Archimedean space under some open surjection [119]. If one omits in (2) the assumption that $Y$ is a monotonically developable then by [37, Corollary $7.2]$ it is possible to guarantee only the existence an USC compactvalued selection $H: X \rightarrow Y$.

If we equip a paracompact domain $X$ in Theorem 49 by a sequence $\left\{X_{n}\right\}$ of its finite-dimensional subspaces $\operatorname{dim} X_{n} \leq n$, then by [36, Corollary 7.7], we always obtain an USC compactvalued selection $H: X \rightarrow Y$ with $|H(x)| \leq$ $n+1, x \in X_{n}$. If we eqip a paracompact domain $X$ by a sequence $\left\{X_{n}\right\}$ of its finite-dimensional subspaces, $\operatorname{dim} X_{n} \leq n$, then we always obtain an USC compactvalued selection $H: X \rightarrow Y$ with $|H(x)| \leq n+1, x \in X_{n}$.

As a rule, all selections in [37] are constructed as a composition of two suitable multivalued mappings. The first one is related to completeness and the other one arises from a system (tree) of various coverings of the domain and their refinements.

Applying the same "trees-sieves" technique in [39] upper semicontinuity of a selection was replaced by closedness of its graph. Below are two typical examples.

Theorem 50 ([39]). For a $T_{1}$-space $X$ the following statements are equivalent:

(1) $X$ is normal;

(2) If $Y$ is a metrizable space and $F: X \rightarrow Y$ is a compactvalued $L S C$ mapping then there are compactvalued mappings $G: X \rightarrow Y$ and $H: X \rightarrow$ $Y$ such that $G(x) \subset H(x) \subset F(x), x \in X, G$ is LSC and the graph of $H$ is a closed subset of $X \times Y$; 
(3) If $Y$ is a metrizable space and $F: X \rightarrow Y$ is a compactvalued LSC mapping then there exists a compactvalued selection of $F$ with a closed graph.

Theorem 51 ([39]). For a $T_{1}$-space $X$ the following statements are equivalent:

(1) $X$ is countably paracompact and normal;

(2) Same as (2) in theorem above, but for closedvalued mappings into a separable range space.

Choban, Mihaylova and Nedev [19] collected various types of selection characterizations of classes of topological spaces formulated in terms of multivalued selections. Recall that the $n$-th image of a set is defined inductively by setting $F^{1}(A)=F(A), F^{n+1}(A)=F\left(F^{-1}\left(F^{n}(A)\right)\right)$, and that the largest image is defined as the union of all $n$-th images, $n \in \mathbb{N}$.

Theorem 52 ([19]). For a $T_{1}$-space $X$ the following statements are equivalent:

(1) $X$ is strongly paracompact (i.e Hausdorff and each open cover admits a star-finite refinement);

(2) For every LSC mapping $F: X \rightarrow Y$ into a discrete space $Y$ there exists a discrete space $Z$, a singlevalued map $g: Z \rightarrow Y$, a LSC mapping $G: X \rightarrow Z$, and an USC finitevalued mapping $H: X \rightarrow Z$ such that $g(G(x)) \subset g(H(x)) \subset F(x), x \in X$, and all sets $H^{\infty}(x)$ are countable;

(3) Same as (2) but without LSC mapping $G$ and without finiteness of the values $H(x)$;

(4) Same as (2) but with a regular $X$, without USC mapping $H$, and with a countable $G^{\infty}(x)$.

For a space $X$, let $c \omega(X)$ denote the cozero dimensional kernel of $X$, i.e. the complement of the union of all open zero-dimensional subsets of $X$.

Theorem 53 ([19]). For a $T_{1}$-space $X$ the following statements are equivalent:

(1) $X$ is strongly paracompact and $c \omega(X)$ is Lindelöf ;

(2) See (2) in previous theorem with $Y=Z$ and $g=\left.i d\right|_{Z}$;

(3) Same as (2) but without LSC mapping G and without finiteness of values $H(x)$;

(4) Same as (2) but with a regular $X$, without USC mapping $H$ and with a countable $G^{\infty}(x)$.

In the next theorem $l(X)$ denotes the Lindelöf number of the space $X$ and singlevalued selections are not assumed to be continuous.

Theorem 54 ([19]). For any regular space $X$ and any cardinal number $\tau$ the following statements are equivalent: 
(1) $l(X) \leq \tau$;

(2) For every LSC closedvalued mapping $F: X \rightarrow Y$ into a complete metrizable space $Y$ there exists a LSC closedvalued selection $G$ of $F$ such that $l(G(X)) \leq \tau$;

(3) For every LSC mapping $F: X \rightarrow Y$ into a complete metrizable space $Y$ there exists a singlevalued selection $g$ of $C l o s(F)$ such that $l(g(X)) \leq \tau$;

(4) For every LSC mapping $F: X \rightarrow Y$ into a discrete space $Y$ there exists a singlevalued selection $g$ of $C l o s(F)$ such that $|g(X)| \leq \tau$;

(5) Every open cover of $X$ admits a refinement of cardinality $\leq \tau$.

Similar characterization was obtained in [19] for the degree of compactness of a space. Gutev and Yamauchi in [46], using once again the "treessieves" technique, presented a generalizations of results [19] to arbitrary complete metric range spaces. For example, in comparison with Theorem 52 they proved the following:

Theorem 55 ([46]). For a $T_{1}$-space $X$ the following statements are equivalent:

(1) $X$ is strongly paracompact;

(2) For every LSC closedvalued mapping $F: X \rightarrow Y$ into a complete metric space $(Y ; \rho)$ there exist a complete ultrametric space $(Z ; d)$, a uniformly continuous map $g: Z \rightarrow Y$, and a USC compactvalued mapping $H: X \rightarrow Z$ such that $g(H(x)) \subset F(x), x \in X$, and the set $H\left(H^{-1}(S)\right)$ is totally $\varepsilon$-bounded whenever $\varepsilon>0$ and $S \subset Z$ is totally $\varepsilon$-bounded;

(3) For every LSC closedvalued mapping $F: X \rightarrow Y$ into a discrete space $Y$ there exist a discrete space $Z$, a singlevalued map $g: Z \rightarrow Y$, and a USC compactvalued mapping $H: X \rightarrow Z$ such that $g(H(x)) \subset F(x), x \in X$ and the set $H\left(H^{-1}(S)\right)$ is finite whenever $S \subset Z$ is finite.

Similarly, [46, Corollaries 6.2 and 6.3] a space $X$ is strongly paracompact and $c \omega(X)$ is Lindelöf (resp., compact) if and only if for every LSC closedvalued mapping $F: X \rightarrow Y$ into a completely metrizable space $Y$ there exists a USC compactvalued selection $H$ of $F$ such that set $H\left(H^{-1}(S)\right)$ is separable (resp., compact), whenever $S \subset Z$ is separable (resp., compact).

Yamauchi [116] gave a selection characterization of the class which unifies compact spaces and finite-dimensional paracompact spaces. A topological space is said to be finitistic (another term is boundedly metacompact) if any of its open covers admits an open refinement of finite order, or equivalently for paracompact spaces, if and only if it contains a compact subset $K$ such that each closed subset of the complement of $K$ is finite-dimensional. Below is a typical statement.

Theorem 56 ([116]). For a $T_{1}$-space $X$ the following statements are equivalent:

(1) $X$ is paracompact and finitistic; 
(2) Each LSC closedvalued mapping $F: X \rightarrow Y$ into a completely metrizable space $Y$ admits a USC compactvalued selection $H: X \rightarrow Y$ of $F$ with the property that for every open cover $\nu$ of $Y$ the exists a natural number $N$ such that every value $H(x), x \in X$, can be covered by some $\nu_{0} \subset \nu$ with $\operatorname{Card}\left(\nu_{0}\right) \leq N$.

Finally, let us mentioned the survey paper by Choban [17] on reduction principles in selection theory. Briefly, he discussed questions concerning extensions of LSC mappings $F$ with nonparacompact domains onto paracompact ones and a notion of (complete) metrizability of family $\{F(x)\}$ of values rather than (complete) metrizability of a range space.

\section{Miscellaneous results}

7.1. Tymchatyn and Zarichnyi [109] applied the selection theorem of Fryszkowski [33] to decomposable-valued mappings $F: X \rightarrow L_{1}([0 ; 1], B)$ in order to construct a continuous linear regular operator which extends partially defined pseudometrics to pseudometrics defined on the whole domain. Denote by $\mathcal{P} \mathcal{M}(X)$ the set of all continuous pseudometrics over metrizable compact $X$ and $\mathcal{P} \mathcal{M}$ the subset of $\mathcal{P} \mathcal{M}(X)$ of all continuous pseudometrics $\rho$ with compact domains $\operatorname{dom}(\rho) \subset X$. Identifying a pseudometric with its graph we can consider both of these sets endowed with the topology induced from the compact exponent $\exp (X \times X \times[0 ; \infty))$.

Theorem 57 ([109]). There exists a continuous linear regular extension operator $u: \mathcal{P} \mathcal{M} \rightarrow \mathcal{P} \mathcal{M}(X),\left.\quad u(\rho)\right|_{\operatorname{dom}(\rho) \times \operatorname{dom}(\rho)} \equiv \rho$.

Here, regularity of an operator means that it preserves the norm of pseudometrics, i.e. their maximal values. As it typically arises for extensions, the answer is given by some formula. Namely, under some isometric embedding $X$ into a separable Banach $B$, the desired operator $u$ can be defined as

$$
u(\rho)(x, y)=\int_{0}^{1} \rho(f(\operatorname{dom}(\rho), x)(t), f(\operatorname{dom}(\rho), y)(t)) d t,
$$

where $f: \exp X \times X \rightarrow L_{1}([0 ; 1], B)$ is a continuous singlevalued selection of the decomposable-valued LSC mapping $F: \exp X \times X \rightarrow L_{1}([0 ; 1], B)$ defined by

$$
F(A, x)=L_{1}([0 ; 1],\{x\})=\{x\}, x \in A ; \quad F(A, x)=L_{1}([0 ; 1], A), x \notin A .
$$

Metrizability of a compact space $X$ is a strongly essential assumption [109, Theorem 6.1].

Theorem 58 ([109]). For a compact Hausdorff space $X$ the following statements are equivalent: 
(1) $X$ is metrizable;

(2) There exists a continuous extension operator $u: \mathcal{P M} \rightarrow \mathcal{P} \mathcal{M}(X)$.

7.2. Gutev and Valov[45] applied selection theory to obtain a new proof of Prokhorov's theorem and its generalization outside the class of Polish spaces. Recall that a probability measure $\mu$ on a $T_{3,5}$-space $X$ is a countably additive mapping $\mu: \mathcal{B}(X) \rightarrow[0,1]$ with $\mu(X)=1$ and with regularity (or, the Radon) property that

$$
\mu(B)=\sup \{\mu(K): K \subset B, K \text { is compact }\}
$$

for every Borel set $B \in \mathcal{B}(X)$. Roughly speaking, values of measure are realized over subcompacta with any precision.

The set $P(X)$ of all probability measures can naturally be considered as the subset of the conjugate space $C^{*}(X)$ of the Banach space $C(X)$ and is endowed with the induced topology. Thus Prokhorov's theorem states that for a Polish space $X$, the Radon property holds not for a unique measure but for an arbitrary compact set of measures. Namely, for a compact $M \subset P(X)$ and for any $\varepsilon>0$, there exists a compact $K \subset X$ such that $\mu(X \backslash K)<\varepsilon$, for all $\mu \in M$. For more general domains the following result holds [45]:

Theorem 59 ([45]). For a sieve-complete space $X$, a paracompact space $S \subset P(X)$ and any $\varepsilon>0$, there exists a USC compactvalued mapping $H$ : $S \rightarrow P(X)$ such that $\mu(X \backslash H(\mu))<\varepsilon$, for every $\mu \in S$.

Note that for a paracompact space $X$, its sieve-completeness coincides with its Czech-completeness. Returning to the original case of Polish space $X$, the outline of the proof looks as follows. First, for each $\mu \in P(X)$ the set $G_{\varepsilon}(\mu)=\{K$ - compact $: \mu(X \backslash K)<0,5 \varepsilon\} \subset P(X)$ is nonempty simply due to the Radon property. Denote by $\exp (X)$ the completely metrizable space of all subcompacta of $X$ endowed with the Vietoris topology, or with Hausdorff distance metric. It turns out that the multivalued mapping $G_{\varepsilon}: P(X) \rightarrow$ $\exp (X)$ is a LSC mapping. Hence its pointwise closure $F_{\varepsilon}$ is also LSC and moreover, $\mu(X \backslash K) \leq 0,5 \varepsilon$, for every $\mu \in P(X)$ and every $K \in F_{\varepsilon}(\mu)$. By the compactvalued selection Theorem 3 , the mapping $F_{\varepsilon}$ admits an USC compactvalued selection, say $H: P(X) \rightarrow \exp (X)$. Finally, the union $\cup\left\{K^{\prime}\right.$ : $\left.K^{\prime} \in H(\mu)\right\}$ yields the desired compact subset $K \subset X$.

7.3. Zippin [123] considered the convexvalued selection Theorem 1 as the base for resolving the extension problem for operators from a linear subspaces $E$ of $c_{0}$ into the spaces $C(K)$, where $K$ is a compact Hausdorff space. For any $\varepsilon>0$, he considered the multivalued mapping $F: \operatorname{Ball}\left(E^{*}\right) \rightarrow(1+\varepsilon) \operatorname{Ball}\left(c_{0}^{*}\right)$ by setting $F\left(e^{*}\right)$ equal to $\{0\}$ if $e^{*}=0$ and

$$
F\left(e^{*}\right)=\left\{x^{*} \in(1+\varepsilon) \operatorname{Ball}\left(c_{0}^{*}\right): x^{*} \text { extends } e^{*} \text { and }\left\|x^{*}\right\|<(1+\varepsilon)\left\|e^{*}\right\|\right\}
$$

otherwise. Under the weak-star topology, all values of $F$ are convex metrizable compacta. After (a nontrivial) verification of lower semicontinuity of $F$ and 
applying Theorem 1, one finds a singlevalued weak-star continuous mapping $f: \operatorname{Ball}(E *) \rightarrow(1+\varepsilon) \operatorname{Ball}\left(c_{0}^{*}\right)$ such that $f\left(e^{*}\right)$ extends $e^{*}$ and $\left\|f\left(e^{*}\right)\right\| \leq$ $(1+\varepsilon)\left\|e^{*}\right\|$.

Hence for an operator $T: E \rightarrow C(K)$ with the norm $\|T\|=1$, let $f_{T}$ : $K \rightarrow \operatorname{Ball}\left(E^{*}\right)$ be defined by $f_{T}(k)(e)=T(e)(k)$, for $k \in K$. Then $f_{T}$ is weak-star continuous and hence the composition $f \circ f_{T}: K \rightarrow(1+\varepsilon) \operatorname{Ball}\left(c_{0}^{*}\right)$ with the above selection $f$ is also continuous. Defining $\widehat{T}: c_{0} \rightarrow C(K)$ by $\widehat{T}(x)(k)=\left(f \circ f_{T}\right)(k)(x), x \in c_{0}$, we see that $\widehat{T}$ extends $T$ because $f\left(e^{*}\right)$ extends $e^{*}, \widehat{T}$ is linear and well-defined, since $\left(f \circ f_{T}\right)(k)$ is a linear functional and $\|\widehat{T}\|=\sup \{\widehat{T}(x)(k):\|x\| \leq 1, k \in K\} \leq \sup \left\{\left\|\left(f \circ f_{T}\right)(k)\right\|\|x\|:\|x\| \leq\right.$ $1, k \in K\} \leq 1+\varepsilon$.

7.4. For two multivalued mappings $F_{1}: X \rightarrow Y_{1}, F_{2}: X \rightarrow Y_{2}$ and a singlevalued mapping $L: Y_{1} \times Y_{2} \rightarrow Y$ denote by $L\left(F_{1} ; F_{2}\right)$ the composite mapping, which associates to each $x \in X$ the set

$$
\left\{y \in Y: y=L\left(y_{1} ; y_{2}\right), y_{1} \in F_{1}(x), y_{2} \in F_{2}(x)\right\} .
$$

Definition 8. Let $f$ be a selection of the composite mapping $L\left(F_{1} ; F_{2}\right)$. Then pair $\left(f_{1}, f_{2}\right)$ is said to be a splitting of $f$ if $f_{1}$ is a selection of $F_{1}, f_{2}$ is a selection of $F_{2}$ and $f=L\left(f_{1} ; f_{2}\right)$.

Therefore the splitting problem [90] for the triple $\left(F_{1}, F_{2}, L\right)$ is the problem of finding continuous selections $f_{1}$ and $f_{2}$ which split a continuous selection $f$ of the composite mapping $L\left(F_{1} ; F_{2}\right)$. As a special case of a constant mappings $F_{1} \equiv A \subset Y, F_{2} \equiv B \subset Y$ and $L\left(x_{1}, x_{2}\right)=x_{1}+x_{2}$ we have the following:

Problem 5 ([90]). Let $A$ and $B$ be closed convex subsets of a Banach space $Y$ and $C=A+B$ their Minkowski sum. Is it possible to find continuous singlevalued mappings $a: C \rightarrow A$ and $b: C \rightarrow B$ such that $c=a(c)+b(c)$ for all $c \in C$ ?

The answer is positive for strictly convex and finite-dimensional $A$ and $B$ (cf. [92, Corollary 3.6]), and for finite-dimensional $A$ and $B$ with $C=$ $A+B$ being of a special kind, the so-called $P$-set (cf. [6, Theorem 2.6]). A collection of various examples and affirmative results on approximative splittings, uniformly continuous (or Lipschitz) splittings can be found in [4, $6,7]$. Note that under the replacement of the sum-operator $L\left(x_{1}, x_{2}\right)=x_{1}+x_{2}$ by an arbitrary linear operator $L$, the problem has a negative solution even in a rather low-dimensional situation [92, Example 3.2].

Theorem 60 ([92]). For any 2-dimensional cell D there exist:

(a) Constant multivalued mappings $F_{1}: D \rightarrow R^{3}$ and $F_{2}: D \rightarrow R$ with convex compact values;

(b) A linear surjection $L: R^{3} \oplus R \rightarrow R^{2}$; and

(c) A continuous selection $f$ of the composite mapping $F=L\left(F_{1}, F_{2}\right)$, such that $f \neq L\left(f_{1}, f_{2}\right)$ for any continuous selections $f_{i}$ of $F_{i}, i=1$,2. 
The construction uses the convex hull $C$ of one full rotation of the spiral $K=\{(\cos t, \sin t, t): 0 \leq t \leq 2 \pi\}$ and the fact that its projection onto the $x y$-plane admits no continuous singlevalued selections.

7.5. As for the finite-dimensional selection Theorem 4, during the discussed period three volumnuous papers were devoted to its versions, generalizations or applications. In [44] Gutev and Valov proved the following result on the density of selections.

Theorem 61 ([44]). Let for a mapping $F: X \rightarrow Y$ all assumptions of Theorem 4 be true. Let in addition $\Psi: X \rightarrow Y$ be a mapping with an $F_{\sigma}$-graph such that for each $x \in X$ the intersection $F(x) \cap \Psi(x)$ is a $\sigma Z_{n+1}$-subset of the value $F(x)$. Then in the set $\operatorname{Sel}(F)$ of all continuous singlevalued selections of $F$ endowed with the fine topology the subset of those selections of $F$ which pointwisely avoid values $\Psi(x)$ constitutes a dense $G_{\delta}$-subspace.

Recall that for a metric range space $(Y ; d)$ the fine topology in $C(X, Y)$ is defined by its local base

$$
V(f, \varepsilon(\cdot))=\{g \in C(X, Y): d(g(x), f(x))<\varepsilon(x), x \in X\},
$$

when $\varepsilon(\cdot)$ runs over the set of all positive continuous functions on $X$. Also, for metric space $(B ; \varrho)$, a subset $A \subset B$ is said to be a $\sigma Z_{n+1}$-subset if it is the union of countably many sets $A_{i} \subset B$ such that each continuous mapping from the $(n+1)$-cell to $B$ can be approximated (with respect to the uniform topology) by a sequence of continuous mappings to $B \backslash A_{i}$. The sequential process of proving this theorem is based on the following result.

Theorem 62 ([44]). Let all assumptions of Theorem 61 be true with exception that $\Psi$ is a closed-graph mapping. Let $f$ be a continuous singlevalued selection of $F, \varepsilon(\cdot)$ a positive continuous function on $X$ and $\varrho$ a compatible metric on $Y$. Then $F$ admits a continuous singlevalued selection $g$ such that $g(x) \notin \Psi(x)$ and $\varrho(g(x), f(x))<\varepsilon(x)$, for every $x \in X$.

Next, recall that Shchepin and Brodsky [101] proved that for any paracompact space $X$ with $\operatorname{dim} X \leq n+1$, a completely metrizable space $Y$, and for any $L$-filtration $\left\{F_{i}\right\}_{i=0}^{n+1}$ of maps $F_{i}: X \rightarrow Y$, the ending mapping $F_{n+1}$ admits a continuous singlevalued selection. One of the points in the definition of $L$-filtration (cf. [88]), is the property that graph-values $\{x\} \times F_{i}(x)$ are closed in some prescribed $G_{\delta}$-subset of the product $X \times Y$. In [36, Corollary 7.10] Gutev proved a generalization of this result to the case when the graph of mapping $F_{n+1}$ is a $G_{\delta}$-subset of $X \times Y$. Roughly speaking, Gutev proposed his own version of the Shchepin-Brodsky $L$-filtrations technique. In particular, he generalized the previous theorem [36, Corollary 7.12].

Theorem 63 ([36]). Theorem 61 is true under the change of the assumption $\left(\{F(x)\}_{x \in X}\right)$ is $\left.E L C^{n}\right)$ with the restriction that $F$ be an $E L C^{n}$-mapping, i.e. $\left.\{x\} \times\{F(x)\}_{x \in X}\right)$ is $E L C^{n}$ in the product $X \times Y$. 
As an example of a result on improvement of near-selections in [36, Corollary 7.15$]$ we quote the following:

Theorem 64 ([36]). Let $X$ be a countably paracompact and normal space, $(Y ; d)$ a complete metric space and $F: X \rightarrow Y$ a Hausdorff continuous closedvalued mapping all of whose values $F(x)$ are uniformly $L C^{n}$-subsets of $Y$. Then for every positive lower semicontinuous numerical function $\varepsilon(\cdot)$ on $X$ there exists a positive lower semicontinuous numerical function $\delta(\cdot)$ on $X$ with the following property:

If $g: X \rightarrow Y$ is a continuous singlevalued $\delta$-selection of $F$ then there exists a continuous singlevalued selection $f$ of $F$ such that $d(g(x), f(x))<$ $\epsilon(x), x \in X$.

Moreover, if all values $F(x)$ are $n$-connected then $F$ admits a continuous singlevalued selection.

During preparation of the previous survey [88], Brodsky, Shchepin and Chigogidze announced results on problem of local triviality of Serre fibrations with two-dimensional fibers. Their paper [13] appeared in 2008.

Theorem 65. Let $p: E \rightarrow B$ be a Serre fibration of a locally connected compact space $E$ onto a compact ANR-space B. Let all fibers $p^{-1}(x)$ be homeomorphic to a fixed two-dimensional manifold $M$ which differs from the sphere and the projective plane. Then $p$ admits a continuous section (i.e. $p^{-1}: B \rightarrow E$ admits a continuous singlevalued selection) provided that one of the following restrictions holds:

(1) $\pi_{1}(M)$ is abelian and $H^{2}\left(B ; \pi_{1}(M)\right)=0$;

(2) $\pi_{1}(M)$ is nonabelian, $M$ is not the Klein bottle and $\pi_{1}(B)=0$;

(3) $M$ is the Klein bottle and $\pi_{1}(B)=\pi_{2}(B)=0$.

The proof follows the strategy of [101] and is based on selection results for $L$-filtrations which, roughly speaking, are realized by the interplay between $L$-theory and $U$-theory of multivalued mappings (cf. [88]).

7.6. Various results exist in continuous selection theory with some additional restriction of algebraic nature. For example, a multivalued mapping $F: X \rightarrow Y$ between (as a rule, locally convex topological) vector spaces is called additive if $F\left(x_{1}+x_{2}\right)=F\left(x_{1}\right)+F\left(x_{2}\right), x_{1}, x_{2} \in X$, i.e., the image of the sum of two points coincides with the Minkowski sum of the images of these points. Next, $F: X \rightarrow Y$ is called subadditive ( resp., superadditive, resp., convex) if $F\left(x_{1}+x_{2}\right) \subset F\left(x_{1}\right)+F\left(x_{2}\right)$, (resp., if $F\left(x_{1}+x_{2}\right) \supset F\left(x_{1}\right)+F\left(x_{2}\right)$, resp., if $\left.F\left(t x_{1}+(1-t) x_{2}\right) \subset t F\left(x_{1}\right)+(1-t) F\left(x_{2}\right), t \in[0,1]\right)$. The existence of linear selections was proved for several such types of mappings with compact convex values in locally convex spaces. In particular, additive mappings always have linear selections [34], and every superadditive mapping possesses a linear selection [108]. Recently, Protasov [82] obtained criteria on $X$ and $Y$ for the affirmative answer on existence of linear selections for arbitrary subadditive mappings. 
Theorem 66. (1) Any subadditive mapping $F: X \rightarrow Y$ with compact convex values has a continuous linear selection if and only if $\operatorname{dim} X=1$ or $\operatorname{dim} Y=1$.

(2) Any convex mapping $F: X \rightarrow Y$ with compact convex values has a continuous affine selection if and only if $\operatorname{dim} Y=1$.

Moreover, in "only if" parts of (1) and (2) one can omit the continuity restriction: if $\operatorname{dim} Y \geq 2$ then there exists a convex mapping $F: X \rightarrow Y$ without affine selections. Applications in Lipschitz stability problem for linear operators in Banach spaces are presented in [82] as well.

7.7. We end our survey by some selected results from metric projection theory. For more detailed information cf. [15, 29].

Let us recall that the operator of almost best approximation, or $\varepsilon$-projection, of a real Banach space $\left(X,\|\cdot\|_{X}\right)$ to a set $M \subset X$ is defined as the multivalued map

$$
x \mapsto P_{M, \varepsilon}(x)=\left\{z \in M:\|z-x\|_{X} \leq \rho(x, M)+\varepsilon\right\},
$$

where $\rho(x, M)=\inf _{y \in M}\|x-y\|_{X}$ is the distance from $x$ to $M$. If $\varepsilon=0$, then $P_{M}=P_{M, 0}$ is the metric projection operator. Clearly, all sets $P_{M, \varepsilon}(x)$ are nonempty, whereas the equality $P_{M}(x)=\emptyset$ is in general, possible.

If $\|z-x\|_{X} \leq \rho(x, M)+\varepsilon$ is replaced by $\|z-x\|_{X} \leq(1+\epsilon) \rho(x, M)$ then such multivalued mapping is called a multiplicative $\varepsilon$-projection onto $M$.

Recall the Konyagin theorem [56] which states that the metric $\varepsilon$-projection operator admits a continuous singlevalued selection in the case $X=C[0 ; 1]$ with the standard norm and where $M=R=\left\{\frac{f}{g}: f \in U, g \in W\right\}$ is the set of all generalized fractions with $U$ and $W$ linear closed subspaces of $X$. Note that this result is not true for $X=L_{p}[0 ; 1]$.

Ryutin $[95,96]$ considered the set

$$
R_{U, W}=\operatorname{Clos}\left\{\frac{f}{g}: f \in U, g \in W \text { and } g>0\right\}
$$

of generalized fractions in the space $X=C(K)$, where $K$ is connected metric compact, or in the the space $X=L_{1}[0 ; 1]$, and with finite-dimensional $U$ and $W$.

Theorem $67([96])$. Let the intersection of the set $R_{U, W}$ with the closed unit ball $D \subset C(K)$ be compact. Then for every $\varepsilon>0$ the multiplicative $\varepsilon$-projection of $D$ onto $R_{U, W}$ admits a uniformly continuous singlevalued selection.

In the space $X=L_{1}[0 ; 1]$ the situation is more complicated. Namely, in [95] a wide class of pairs $(U, W)$ of finite-dimensional subspaces in $L_{1}[0 ; 1]$ were found with the property that there exists $\varepsilon_{0}=\varepsilon_{0}(U, W)>0$ such that the multiplicative $\varepsilon$-projection onto $R_{U, W}$ admits a singlevalued continuous selection only if $\varepsilon \geq \varepsilon_{0}$. 
Livshits $[60,61]$ considered $X=C[0 ; 1]$ with the standard norm and continuous selections of the metric $\varepsilon$-projection operator onto the set of all splines (i.e. piecewise polynomials) with nonfixed nodes. Namely, for a fixed $n, d \in \mathbb{N}$, denote by $S_{n}^{d}[0,1]$ the set of all functions $f \in C[0 ; 1]$ such that for some (depending on $f$ ) nodes $0=x_{0}<x_{1}<\cdots<x_{n-1}<x_{n}=1$, each restriction $\left.f\right|_{\left[x_{k-1}, x_{k}\right]}$ is a polynomial of degree $\leq d$.

\section{Theorem $68([60,61])$.}

(1) A continuous singlevalued selection of the metric projection onto the set $S_{n}^{1}[0,1]$ exists if and only if $n \leq 2$;

(2) For any $\varepsilon>0$ and any $n \in \mathbb{N}$, there exists a continuous singlevalued selection of the metric $\varepsilon$-projection onto the set $S_{n}^{1}[0,1]$;

(3) For any $n>1$ and any $d>1$ there exists $\varepsilon=\varepsilon(n, d)$ such that there is no continuous singlevalued selection of the metric $\varepsilon$-projection onto the set $S_{n}^{d}[0,1]$.

\section{Acknowledgements}

The authors were supported by the SRA grants P1-0292-0101, J1-2057-0101 and J1-4144-0101, and the RFBR grant 11-01-00822.

\section{References}

1. Alleche, B., Calbrix, J.: Semi-continuous multifunctions and bases of countable order. Topology Appl. 104:1-3, 3-12 (2000)

2. Arvanitakis, A.: A simultaneous extension theorem. Preprint (2010)

3. Askoy, A., Khamsi, M.: A selection theorem in metric trees. Proc. Amer. Math. Soc. 134:10, 2957-2966 (2006)

4. Balashov, M.: About P-property of convex compacta. Math. Notes 71:3, 295-304 (2002).

5. Balashov, M., Ivanov, G.: Properties of metric projections and parametrization of set-valued mappings with weakly convex values. Math. Notes 80:4, 461-4678 (2006)

6. Balashov, M., Repovš, D.: On the splitting problem for selections. J. Math. Anal. Appl. 355, 277-287 (2009)

7. Balashov, M., Repovš, D.: Uniform convexity and the splitting problem for selections. J. Math. Anal. Appl. 360, 307-316 (2009).

8. Banakh, T., Cauty, R.: A homological selection theorem implying a division theorem for Q-manifolds. Banach Center Publ. 77, 11-22 (2007).

9. Ben-El-Mechaiekh, H., Kryszewski, W.: Equilibria of set-valued maps on nonconvex domains. Trans. Amer. Math. Soc. 349, 4159-4179 (1997)

10. Bessaga C., Pelczynski, A.: Selected Topics in Infinite-Dimensional Topology. Monogr. Mat. 58, PWN, Warsaw (1975)

11. Bielawski, R.: Simplicial convexity and its applications. J. Math. Anal. Appl. 106, 155-171 (1986) 
12. Blum, I., Swaminatham, R.: Continuous selections and realcompactness. Pacific J. Math. 93, 251-260 (1981)

13. Brodsky, N., Shchepin, E., Chigogidze, A.: Sections of Serre fibrations with 2-manifold fibers. Topology Appl. 155, 773-782 (2008)

14. Brown, A.: Set-valued mappings, continuous selections and metric projection. J. Appr. Theory 57, 48-68 (1989)

15. Brown A. L., Deutsch, F., Indumathi, V., Kenderov, P.: Lower semicontinuity concepts, continuous selections and set-valued metric projections. J. Approx. Theory 115, 120$143(2002)$

16. Choban, M. M.: General selection theorems and their applications. (in Russian): Serdica 4, 74-90 (1978)

17. Choban, M.: Reduction principles in the theory of selections. Topology Appl. 155, 787-796 (2008)

18. Choban, M., P. Kenderov, Revalski, J.: Densely defined selections of multivalued mappings. Trans. Amer. Math. Soc. 344, 533-552 (1994)

19. Choban, M., Mihailova, E., Nedev, S.: On selections and classes of spaces. . Topology Appl. 155, 797-804 (2008)

20. Corson, H., Lindenstrauss, J.: Continuous selections with non-metrizable ranges. Trans. Amer. Math. Soc. 121, 492-504(1966)

21. De Blasi, F., Gorniewicz, L., Pianigiani, G.: Extension theorems and topological essentiality in $\alpha$-weakly convex metric spaces. Topol. Methods Nonlinear Anal. 34, 141-160 (2009)

22. De Blasi, F., Pianigiani, G.: Continuous selections in $\alpha$-convex metric spaces. Bull. Pol. Acad. Sci. Math. 52:3, 303-317 (2004)

23. De Blasi, F., Pianigiani, G.: On Michael's selection theorem in compact convex metric spaces. Bull. Cal. Math. Soc. 97, 117-130 (2005)

24. Deutsch, F., Kenderov, P.: Continuous selections and approximate selection for setvalued mappings and applications to metric projections. SIAM J. Math. Anal. 121, 185-194 (1983)

25. Ding, X.: Continuous selections and coincidence theorems on product $G$-convex spaces. Fixed Point Theory Appl. 7, 69-80 (2006)

26. Dobrowolski, T.: On extending mappings into nonlocally convex metric spaces. Proc. Amer. Math. Soc. 93:3, 555-560 (1995)

27. Dobrowolski, T., van Mill, J.: Selections and near-selections in linear spaces without local convexity Fund. Math. 192:3, 215-232 (2006)

28. Dzedzej, Z., Kryszewski, W.: Selections and approximations of convex-valued equivariant mappings. Topol. Meth. Nonlin. Anal., to appear.

29. Espinola, R., Lopez, G.: A survey on nonexpansive selection of metric projection. Proc. Int. Conf. on Fixed Point Th. and Appl., Valencia, Spain, 53-70 (2003)

30. Fillipov, V. V.: On a question of E. A. Michael. Comm. Math. Univ. Carol. 45, 735-737 (2004)

31. Fillipov, V. V.: On a problem of E.Michael. Math. Notes 78:4, 638-640 (2005)

32. Fillipov, V. V., Geintz, V.L.: On convex hulls of compact sets of probability measures with countable supports. Funct. Anal. Appl. 45:1, 69-72 (2011)

33. Fryszkowski, A.: Continuous selections for a class of non-convex multivalued maps. Studia Math. 76, 163-174 (1983)

34. Gorokhovik, V.: Representations of affine multifunctions by affine selections SetValued Anal. 16:2-3, 185-198 (2008)

35. Gutev, V.: Continuous selections, $G_{\delta}$-subsets of Banach spaces and upper semicontinuous mappings. Comm. Math. Univ. Carol. 35, 533-538 (1994)

36. Gutev, V.: Selections and approximations in finite-dimensional spaces. Topology Appl. 146-147, 353-383 (2005)

37. Gutev, V.: Completeness, sections and selections. Set Valued Anal. 15, 275-295(2007)

38. Gutev, V.: Selections and topological convexity. Topology Appl. 155, 814-823 (2008) 
39. Gutev, V.: Closed graph multi-selections. Fund. Math. 211, 85-99 (2011)

40. Gutev, V., Makala, N.: Selections, extensions and collectionwise normality. J. Math. Anal. and Appl. 368:2, 573-577 (2010)

41. Gutev, V. G., Ohta, H., Yamazaki, K.: Selections and sandwich-like properties via semicontinuous Banach-valued functions. J. Math. Soc. Japan 55(2), 499-521 (2003)

42. Gutev, V. G., Ohta, H., Yamazaki, K.: Extensions by means of expansions and selections. Set-Valued Analysis 11, 69-104 (2006)

43. Gutev, V. G., Valov, V.: Continuous selections and $C$-spaces. Proc. Amer. Math. Soc. 130, 233-242 (2002)

44. Gutev, V. G., Valov, V.: Dense families of selections and finite-dimensional spaces. Set-Valued Anal. 11, 373-394 (2003)

45. Gutev, V. G., Valov, V.: Sections, selections and Prokhorov's theorem. J. Math. Anal. Appl. 360:2, 377-379 (2009)

46. Gutev, V., Yamauchi, T.: Strong paracompactness and multi-selections. Topology Appl. 157, 1430-1438 (2010)

47. Horvath, Ch.: Contractibility and generalized convexity. J. Math. Anal. Appl. 156, 341-357 (1991)

48. Horvath, Ch.: Topological convexities, selections and fixed points. Topology Appl. 155, 830-850 (2008)

49. Ivanov, G.: Weak convexity in the senses of Vial and Efimov-Stechkin. Izv. Math. RAN 69:6, 1113-1135 (2005)

50. Ivanov, G.: Continuous selections of multifunctions with weakly convex values. Topology Appl. 155, 851-857 (2008)

51. Ivanšić, I., Rubin, L.: A selections theorem for simplex-valued mappings. Glasnik Mat. 39, 331-333 (2004)

52. Jamison, R. E.: A general theory of convexity. Dissertation, Univ. of Washington, Seattle (1974)

53. Karassev, A.: Michael's problem and weakly infinite-dimensional spaces. Topology Appl. 158:15, 1694-1698 (2008)

54. Khamsi, M., Kirk, W., Yanez, K.: Fixed points and selection theorems in hyperconvex spaces. Proc. Amer. Math. Soc. 128:11, 3275-3283 (2000)

55. Kisielewicz, A.: Continuous selections for nonlower semicontinuous multifunctions. J. Math. Anal. Appl. 286, 160-167 (2003)

56. Konyagin, S.: On continuous operator of generalized rational approximation. Math. Notes 44:3, 404 (1988)

57. Kowalska, A.: Continuous selections and approximations in $\alpha$-convex metric spaces. Disscus. Math. (Diff. Incl. Control and Opt.) 27, 265-294 (2007)

58. Levy, F.: On Helly's theorem and the axioms of convexity. J. Indian Math. Soc. 15, 65-76 (1951)

59. Li, G., H.Li.: Continuous selection theorems and fixed point theorems for fuzzy mappings in $L$-convex spaces. JP J. Fixed Point Theory Appl. 1, 109-120 (2006)

60. Livshits, E.: Continuous almost best approximations in $\mathrm{C}[0,1]$. Funct. Anal. Appl. 35:1, 85-87 (2001)

61. Livshits, E.: Stability of the operator of $\varepsilon$-projection to the set of splines in $\mathrm{C}[0,1]$. Izv. RAN. Ser. Mat. 67:1, 99 - 130 (2003)

62. Mägerl, G.: An unified approach to measurable and continuous selections. Trans. Amer. Math. Soc. 245, 443-452 (1978)

63. Makala, N.: Selections for paraconvexvalued mappings on non-paracompact domains. Topology Appl. 159:2, 153-157 (2012)

64. Markin, J.: A selection theorem for quasi-lower semicontinuous mappings in hyperconvex spaces. J. Math. Anal. Appl. 321, 862-866 (2006)

65. Markin, J.: Fixed points, selections and best approximation for multivalued maps into $R$-trees. Nonlinear Analysis 67, 2712-2716 (2007)

66. Menger, K.: Untersuchungen uber allgemeine Metrik. I, II, III. Math. Ann. 100, 75-163 (1928) 
67. Michael, E. A.: Continuous selections, I. Ann. of Math. 63, 361-382 (1956)

68. Michael, E. A.: Continuous selections, II. Ann. of Math. 64, 562-580 (1956)

69. Michael, E. A.: Dense familiers of continuous selections. Fund. Math. 47, 173-178 (1959)

70. Michael, E. A.: Convex structures and continuous selections. Can. J. Math. 11, 556575 (1959)

71. Michael, E. A.: Paraconvex sets. Math. Scand. 7, 372-376 (1959)

72. Michael, E. A.: A theorem on semi-continuous set-valued functions. Duke Math. J. 26, 647-651 (1959)

73. Michael, E. A.: Continuous selections and countable sets. Fund. Math. 111, 1-10 (1981)

74. Michael, E. A.: Some problems. in Open Problems in Topology, North Holland, Amsterdam, 271-278, (1990)

75. Michael, E. A.: Continuous selections and complete metrizability of the range. J. Fixed Points Theory Appl. 8, 75-79 (2010)

76. Michael, E. A.: A note on a set-valued extension property. Topology Appl. 158, 1526-1528 (2011)

77. Michael, E. A., Namioka I.: A note on convex $G_{\delta}$-subsets of Banach spaces. Topology Appl. 155, 858-860 (2008)

78. van Mill, J.: Infinite-Dimensional Topology: Prerequisites and Introduction. North Holland, Amsterdam, (1989)

79. van Mill, J., Pelant, J., Pol, R.: Selections that characterize topological completeness. Fund. Math. 149, 127-141 (1996)

80. Nedev, S. Y.: A selection example. C. R. Acad. Bulg. Sci. 40:11, 13-14 (1987)

81. Nedev, S., Valov, V.: On metrizability of selectors. C. R. Acad. Bulgare 36 (11), 1363-1366 (1983)

82. Protasov, V.,: On linear selections of convex set-valued maps. Funct. Anal. and Appl. 45:1, 56-68 (2011)

83. Przymusinski, T.,: Collectionwise normality and absolute retracts. Fund. Math. 98, 61-73 (1978)

84. Repovš, D., Semenov, P. V.: On nonconvexity of graphs of polynomials of several real variables. Set-Valued. Anal. 6, 39-60 (1998)

85. Repovš, D., Semenov, P. V.: Continuous Selections of Multivalued Mappings. Math. and Its Appl. 455, Kluwer, Dordrecht (1998)

86. Repovš, D., Semenov, P. V.: Continuous selections as uniform limits of $\delta$-continuous $\varepsilon$-selections. Set-Valued Anal. 7, 239-254 (1999)

87. Repovš, D., Semenov, P. V.: On a relation between nonconvexity of the set and nonconvexity of its $\varepsilon$-neighborhood Matem. Zam. 70:2, 246-259 (2001) (in Russian); Engl. transl.: Math. Notes 70, 221-232 (2001)

88. Repovš, D., Semenov, P. V.: Continuous Selections of Multivalued Mappings. in Recent Progress in Gen. Topology-II., 455, Elsevier Science B.V., Amsterdam, 423-461 (2002)

89. Repovš, D., Semenov, P. V.: A minimax theorem for functions with possibly nonconnected intersections of sublevel sets. J. Math. Anal. Appl. 314, 537-545, (2006)

90. Repovš, D., Semenov, P. V.: Sections of convex bodies and splitting problem for selections. J. Math. Anal. Appl. 334:1, 646-655 (2007)

91. Repovš, D., Semenov, P. V.: On closedeness assumptions in selection theorems. Topology Appl. 154:10, 2185-2195 (2007)

92. Repovš, D., Semenov, P. V.: Hereditary invertible surjections and splitting problems for selections. Topology Appl. 156, 1192-1198 (2009)

93. Repovš, D., Semenov, P. V.: On continuous choice of retractions onto a nonconvex sets. Topology Appl. 157, 1510-1517 (2010)

94. Repovš, D., Semenov, P. V., Shchepin, E. V.: On zero-dimensional Milyutin maps and Michael selection theorem. Topology Appl. 54, 77-83 (1993)

95. Ryutin, K.: On continuity of generalized rational approximation operator in the space $L_{1}[0 ; 1]$. Math. Notes 73:1, 148-153 (2003) 
96. Ryutin, K.: On an uniformly continuous operators of almost best generalized rational approximation. Math. Notes 87:1, 147-150 (2010)

97. Sánchez-Gabites, J. J., Sanjurjo, J. M. R.: Multivalued maps, selections and dynamical systems. Topology Appl. 155, 874-882 (2008).

98. Saveliev, P.: Fixed points and selections of set-valued maps on spaces with convexity. Int. J. Math. and Math. Sci. 24, 595-612 (2000)

99. Semenov, P.: Functionally paraconvex sets. Math. Notes 54, 1236-1240 (1993)

100. Semenov, P. V.: Nonconvexity in problems of multivalued calculus. J. Math. Sci. (N. Y.) 100, 2682-2699 (2000)

101. Shchepin, E., Brodsky, N.: Selections of filtered multivalued mappings. Proc. Steklov Inst. Math. 212, 218-239 (1996)

102. Shishkov, I.: Selections of LSC mappings into Hilbert spaces. C.R. Acad. Bulg. Sci. 53:7, 5-8 (2000)

103. Shishkov, I.: $\sigma$-products and selections of set-valued mappings. Comment. Math. Univ. Carol. 42, 203-207 (2001)

104. Shishkov, I.: Extensions of 1.s.c. mappings into reflexive Banach spaces. Set-Valued Anal. 10, 79-87 (2002)

105. Shishkov, I.: Selections of set-valued mappings with hereditary collectionwise domains. Topology Appl. 124, 95-100, (2004)

106. Shishkov, I.: c-Paracompactness and selections. Mat. Balk. 21, 51-57 (2007)

107. Shishkov, I.: Collectionwise normality and selections into Hilbert spaces. Topology Appl. 155, 889-897 (2008)

108. Smajdor, A., Smajdor, W.: Affine selections of convex set-valued functions Aequationes Math. 51:1-2, 12-20 (1996)

109. Tymchatyn, E., Zarichnyi, M.: On simultaneous extension of partial (pseudo)metrics. Proc. Amer. Math. Soc. 132:9, 2799-2807 (2004)

110. Uspenskii, V. V.: A selection theorem for C-spaces. Topol. Appl. 85, 351-374 (1998)

111. Valov, V.: On a theorem of Arvanitakis. Preprint. http://arXiv:1108.2695 (2011)

112. van de Vel, M.: A selection theorem for topological convex structures. Trans. Amer. Math. Soc. 336, 463-496 (1993)

113. Vial, J.: Strong and weak convexity of sets and functions. Math. Oper. Res. 8:2, 231-259 (1989)

114. Yamauchi, T.: On a selection theorem of Blum and Swaminatham. Comment. Math. Univ. Carolin. 45:4, 681-691 (2004)

115. Yamauchi, T.: Continuous selections of symplex-valued mappings. Sci. Math. Japonicae 63:2, 183-189 (2006)

116. Yamauchi, T.: Characterizations of finitistic spaces. Houston J. Math. 33:3, 735-751 (2007)

117. Yamauchi, T.: Continuous selections avoiding extreme points. Topology Appl. 155, 916-922 (2008)

118. Yang, M-G., Deng, L.: Continuous selection theorem for $\Phi_{F C}$-mappings in $F C$-spaces and its applications. Nonlinear Funct. Anal. Appl. 13:4, 563-572 (2008)

119. Wicke, H.: The regular open continuous images of complete metric spaces. Pacific J. Math. 23, 621-625 (1967)

120. Wikipedia: http://en.wikipedia.org/wiki/Tropical_geometry.

121. Wu, X.: Michael selection theorem in hyperconvex metric spaces. Z. für Analysis und ihre Appl. 22:3, 505-516 (2003)

122. Zarichnyi, M.: Michael selection theorem for max-plus compact convex sets. Topology Proc. 31, 677-681 (2007)

123. Zippin, M.: Application of Michael's selection theorem to operator extension problems. Proc. Amer. Math. Soc. 127:5, 1371-1379 (1999) 